Supporting Information

\title{
Synthesis and Evaluation of \\ 3-(Carboxymethylidene)- and 3-(Carboxymethyl)penicillinates as Inhibitors of ㅁ-Lactamase
}

John D. Buynak,* Venkat Rao Ghadachanda, Lakshminaryana Vogeti, Hongming Zhang,

$$
\text { and Hansong Chen }
$$

Department of Chemistry, Southern Methodist University, Dallas, Texas 75275-0314

jbuynak@smu.edu 


\section{Table of Contents}

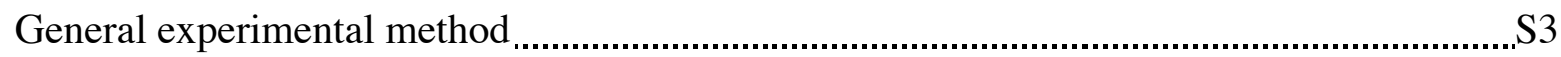

Procedure for Preparation of 6,6-Dibromopenicillanic Acid (6)........................................S3

Procedure for preparation of Penicillanic Acid S,S-Dioxide (7) ...........................................S4

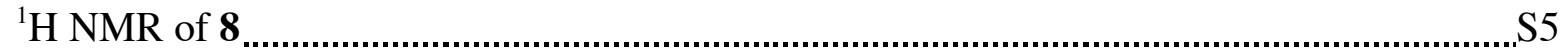

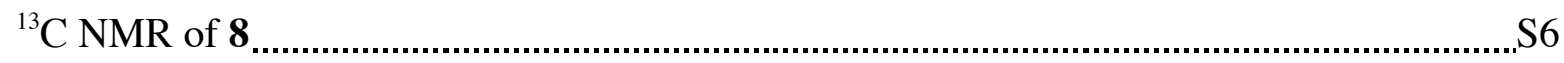

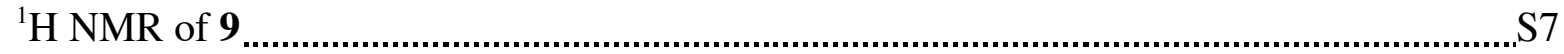

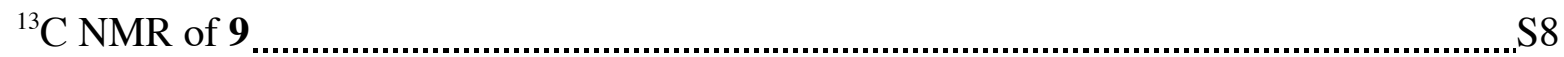

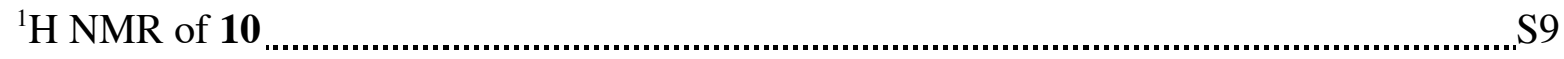

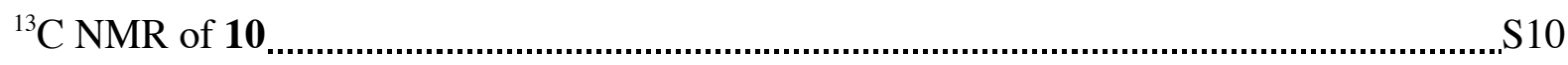

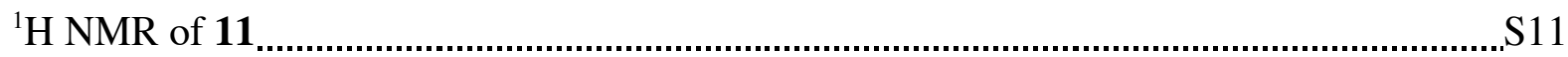

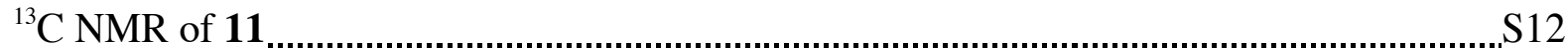

${ }^{1} \mathrm{H}$ NMR OF 12

${ }^{13}$ C NMR of 12

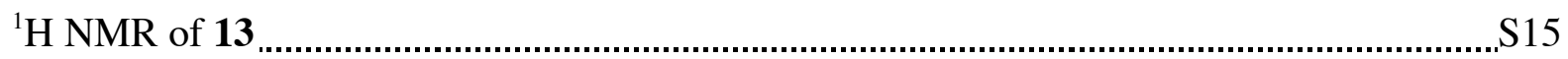

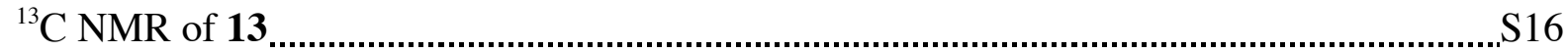

${ }^{1}$ H NMR of 14

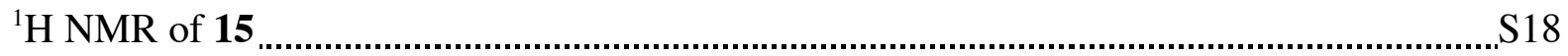

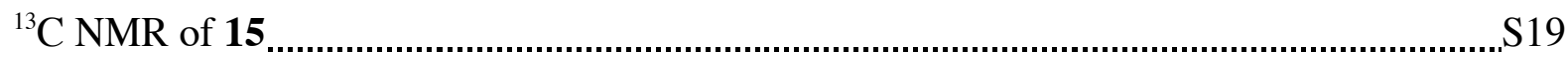

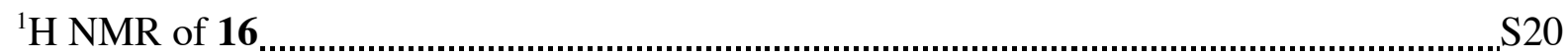

${ }^{13} \mathrm{C}$ NMR of 16

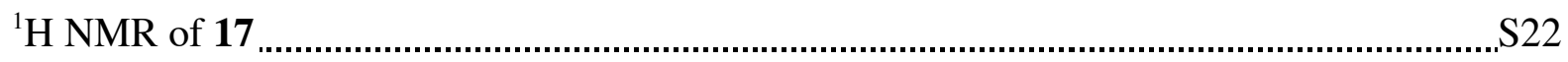

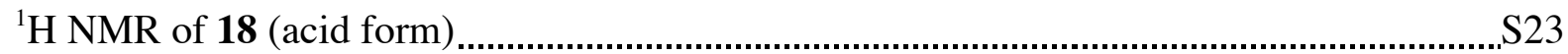

${ }^{1}$ H NMR of $\mathbf{1 8}$ (sodium salt) ......................................................................................... 24

${ }^{1}$ H NMR of 19

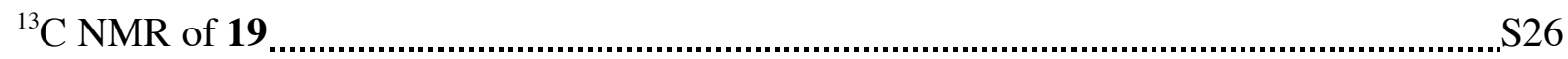

${ }^{1}$ H NMR of 20 (acid form) .............................................................................................. 227

${ }^{1} \mathrm{H}$ NMR of 20 (sodium salt) ............................................................................................. 228

Table 1. Crystal data and structure refinement for compound 9......................................S29

Table 2. Atomic coordinates and equivalent isotropic displacement parameters for 9........S30

Table 3. Bond lengths $[\approx]$ and angles $[\infty]$ for 9 ............................................................S31

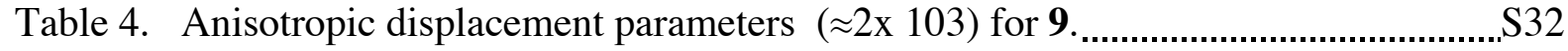

Table 5. Hydrogen coordinates and isotropic displacement parameters for 9.....................S33

Table 6. Crystal data and structure refinement for compound 16.....................................S34

Table 7. Atomic coordinates and equivalent isotropic displacement parameters for $\mathbf{1 6}$.......S35

Table 8. Bond lengths and anglesfor 16..................................................................S36

Table 9. Anisotropic displacement parameters for 16..................................................S38

Table 10. Hydrogen coordinates and isotropic displacement parameters for 16.................S39 


\section{General experimental information}

Unless otherwise noted, all reactions were carried out under $\mathrm{N}_{2}$ atmosphere in oven-dried glassware. Reagents were used as supplied from commercial sources without further purification. 6,6-Dibromopenicillinic acid S, S-dioxide, penicillanic acid S,S-dioxide and diazomethane were prepared according to literature procedures. Flash chromatography was carried out on silica gel 60 (230-400 mesh). NMR spectra were recorded on a $400 \mathrm{MHz} \mathrm{NMR}$ spectrometer using $\mathrm{CDCl}_{3}$ as solvent. Chemical shifts and coupling constants are given in ppm and $\mathrm{Hz}$, respectively.

6,6-Dibromopenicallinic Acid (6): To a 3 L three-necked round-bottomed flask containing methylene chloride $(500 \mathrm{~mL})$ cooled to $5{ }^{\circ} \mathrm{C}$ were added bromine $(119.9 \mathrm{~g}, 38.5 \mathrm{~mL}, 0.75$ $\mathrm{mol}), 2.5 \mathrm{~N}$ sulfuric acid $(200 \mathrm{~mL})$, and sodium nitrite $(34.5 \mathrm{~g}, 0.50 \mathrm{~mol})$. Then 6-APA $(54.0 \mathrm{~g}$ .025 mole) was added portion-wise over a period of $30 \mathrm{~min}$. The resultant dark red solution was stirred at $5{ }^{\circ} \mathrm{C}$ for $30 \mathrm{~min}$. A solution of $1 \mathrm{M}$ sodium bisulfite $(410 \mathrm{~mL})$ was added slowly until the bromine color was discharged, forming a light yellow solution. The organic layer was separated and the aqueous layer extracted with methylene chloride. The combined organic layers were washed with brine and used in the next step.

To a $4 \mathrm{~L}$ beaker containing a methylene chloride solution from first step (900 mL) was added water $(300 \mathrm{~mL})$ followed by the drop-wise addition over a period of $30 \mathrm{~min}$ of $3 \mathrm{~N}$ $\mathrm{NaOH}(105 \mathrm{~mL})$ until the $\mathrm{pH}$ stabilized at 7.0. The aqueous layer was separated, and the organic layer was again extracted with water. The combined aqueous layers were placed in a $2 \mathrm{~L}$ three-necked round bottom flask equipped with mechanical stirrer and cooled to $-5{ }^{\circ} \mathrm{C}$. Then was added a premixed solution containing potassium permanganate $(59.25 \mathrm{~g}, 0.38 \mathrm{~mol})$, 
$85 \%$ phosphoric acid $(18 \mathrm{~mL}, 0.31 \mathrm{~mol})$, and water $(600 \mathrm{~mL})$ over a period of $50 \mathrm{~min}$ until the oxidation was complete as indicated by the persistence of the dark purple permanganate color. The $\mathrm{pH}$ of the purple solution was lowered to 1.23 with $6 \mathrm{~N} \mathrm{HCl}$. To this biphasic solution was added $1 \mathrm{M}$-sodium bisulfite solutions as the temperature was kept below $10{ }^{\circ} \mathrm{C}$ and the $\mathrm{pH}$ was maintained at $1.25-1.35$ by using $6 \mathrm{~N}$ hydrochloric acid. The aqueous solution was extracted with EtOAc and the combined organic extracts were washed with brine, dried over the sodium sulfate, and the solvent was removed under reduced pressure to give the 6,6dibromopenicillanic acid. ${ }^{1} \mathrm{H}$ NMR $\left(400 \mathrm{MHz} \mathrm{CDCl}_{3}\right) \square 5.39$ (s, 1H), 4.62 (s, 1H), 1.57 (s, 1H), 1.63 (s, 3H) $1.44(\mathrm{~s}, 3 \mathrm{H})$.

Penicillanic Acid $S, S$-Dioxide (7): To a 3L three-necked round bottomed flask containing 6,6-dibromopencillinic acid S,S-dioxide (60 g, $149 \mathrm{mmol})$ was added ethyl acetate (1000 $\mathrm{mL})$ and water $(300 \mathrm{~mL})$. The resultant solution was cooled to about $5{ }^{\circ} \mathrm{C}$, and while maintaining the $\mathrm{pH}$ at about 3.5 with $4 \mathrm{~N} \mathrm{HCl}$, magnesium powder (38 g) was added slowly over period of $50 \mathrm{~min}$. The reaction mixture was then stirred at $\mathrm{rt}$ for $10 \mathrm{~h}$. After completion of the reaction, the aqueous layer was extracted with additional ethyl acetate and the extract was washed with brine, dried over sodium sulfate and the solvent was removed under reduced pressure to produce $95 \%$ pure sulbactam. ${ }^{1} \mathrm{H}$ NMR $\left(400 \mathrm{MHz} \mathrm{CDCl}_{3}\right) \square 4.64-4.62(\mathrm{~m}, 1 \mathrm{H}), 4.42(\mathrm{~s}, 1 \mathrm{H})$, 3.50-3.48 (m, 2H), $1.65(\mathrm{~s}, 3 \mathrm{H}) 1.50(\mathrm{~s}, 3 \mathrm{H})$. 


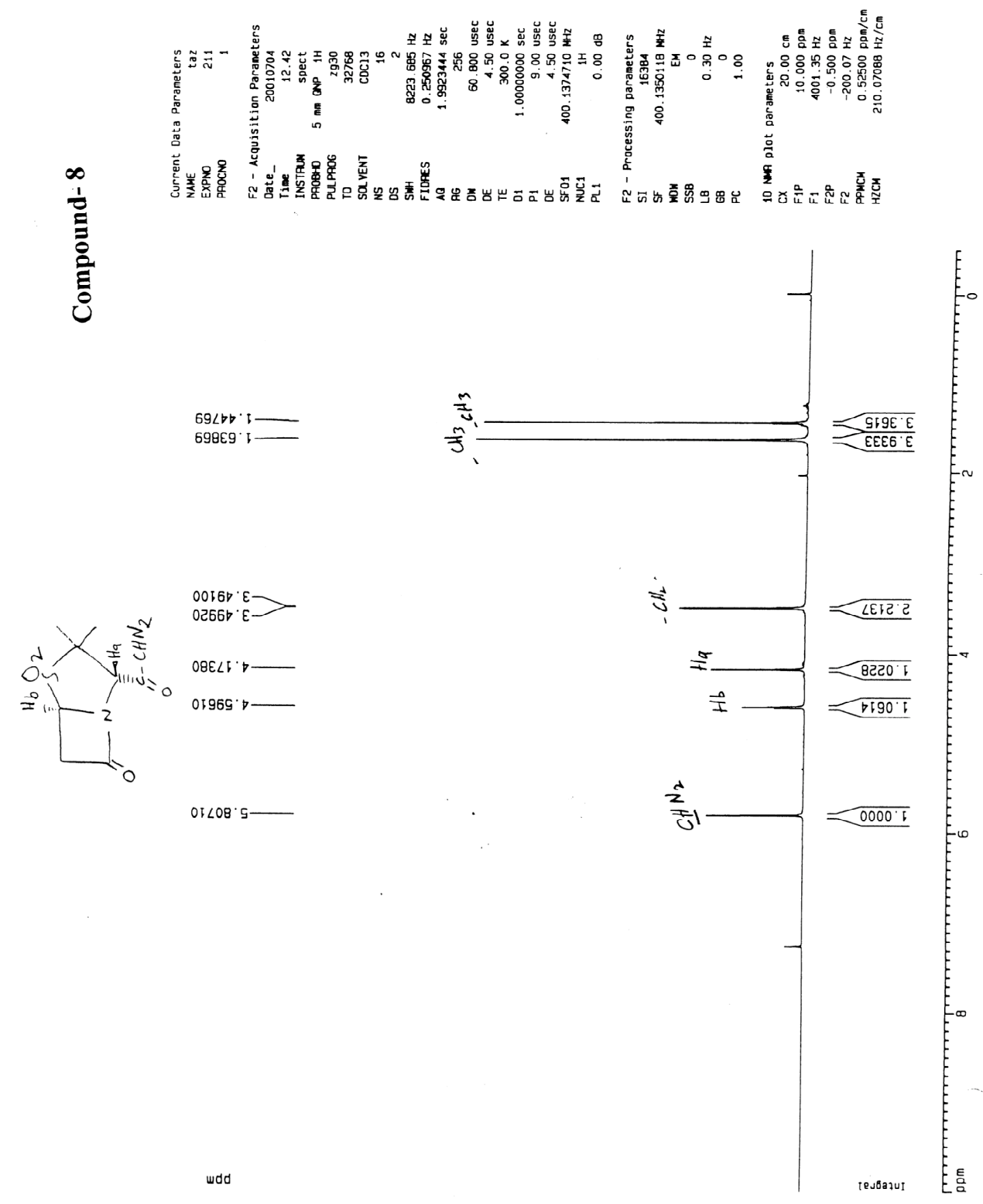



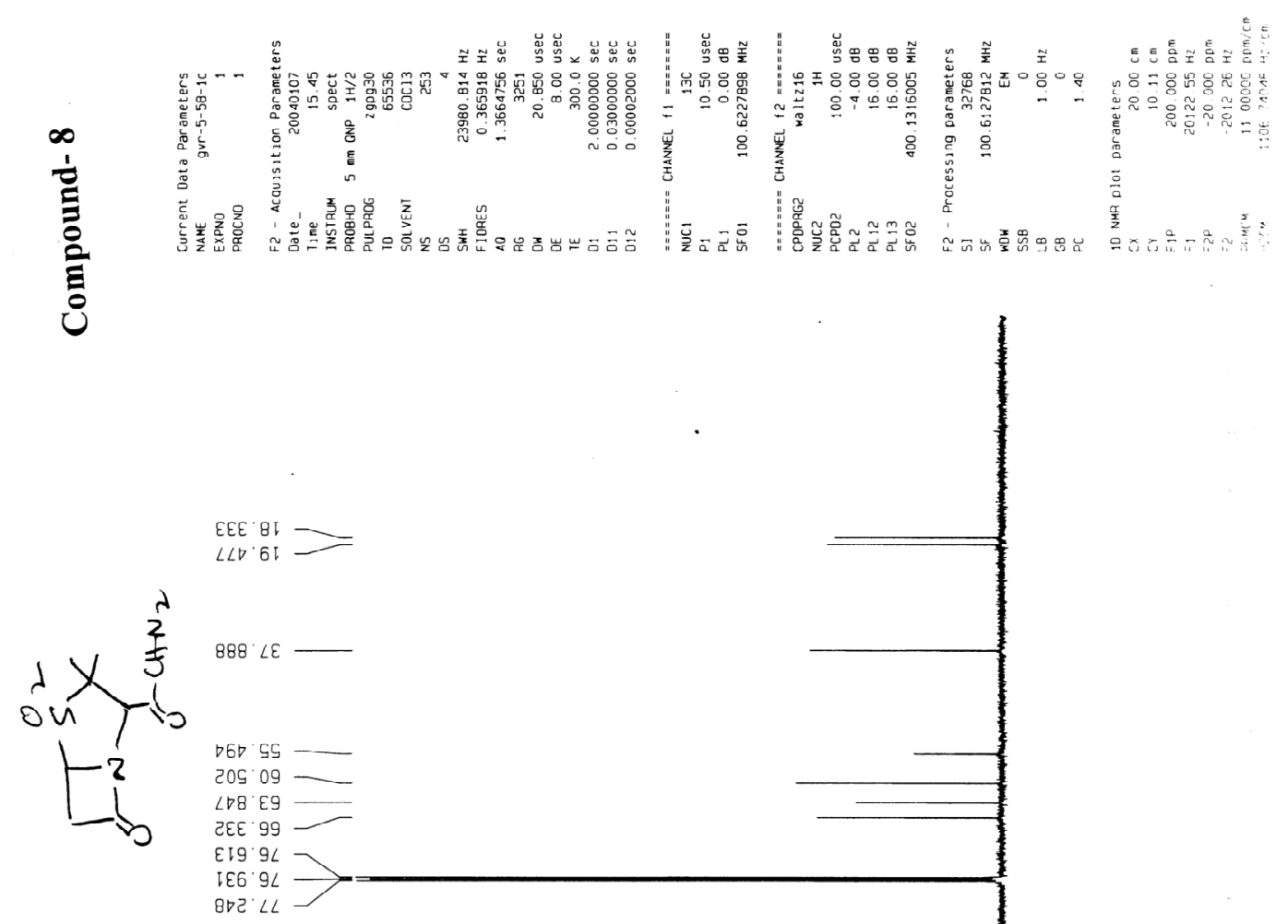

$2 L L L L$

เ68.88I-

wdd 


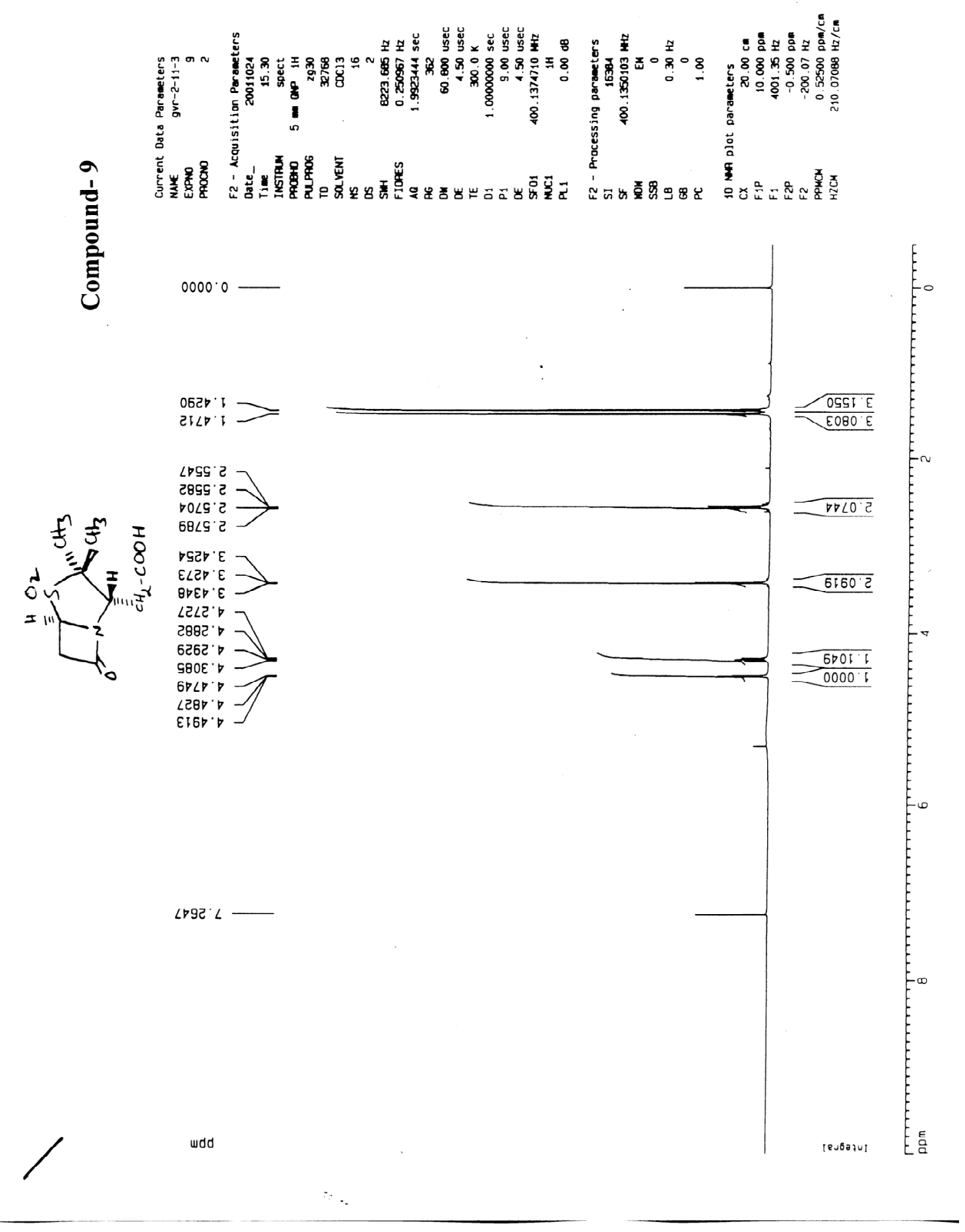




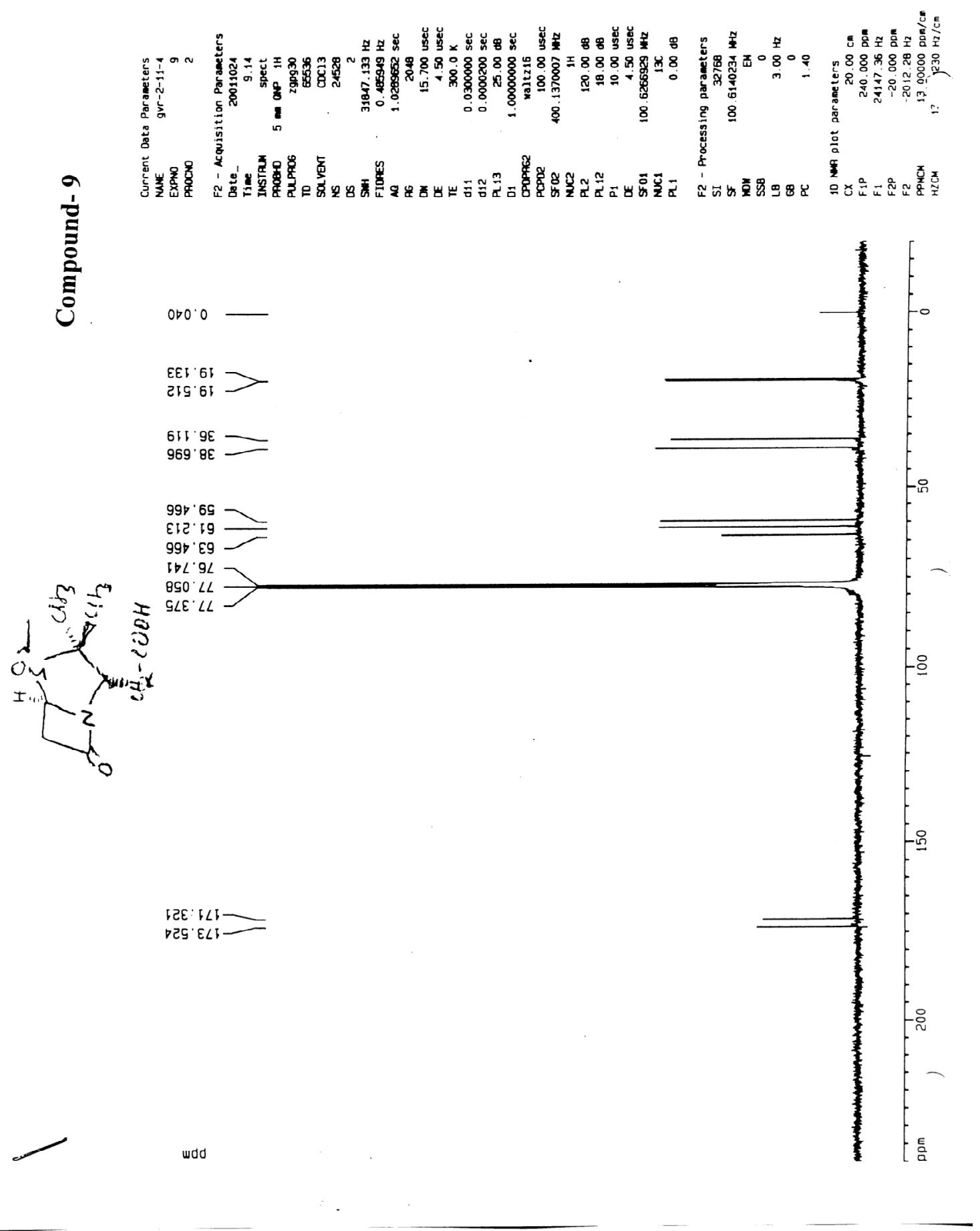




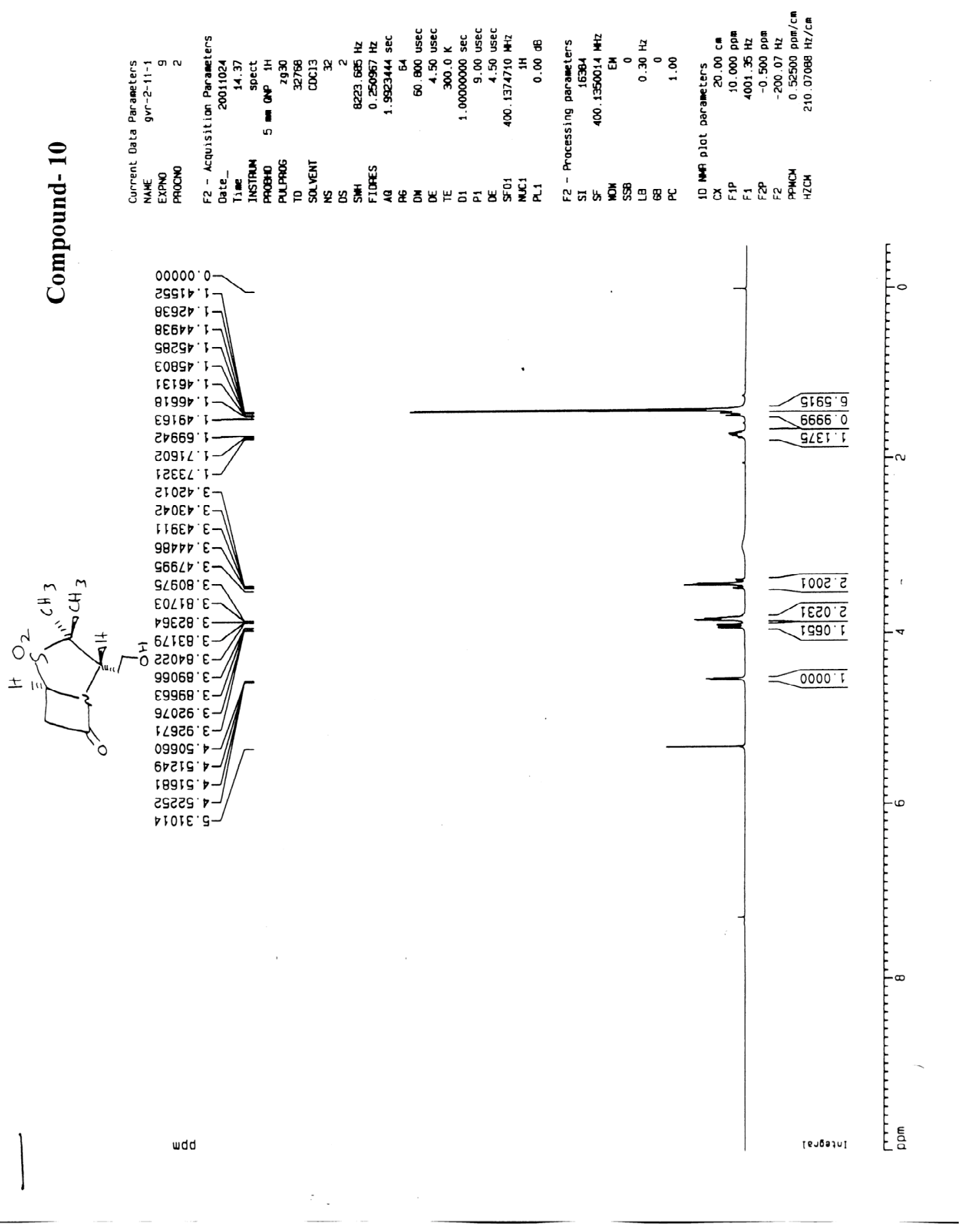




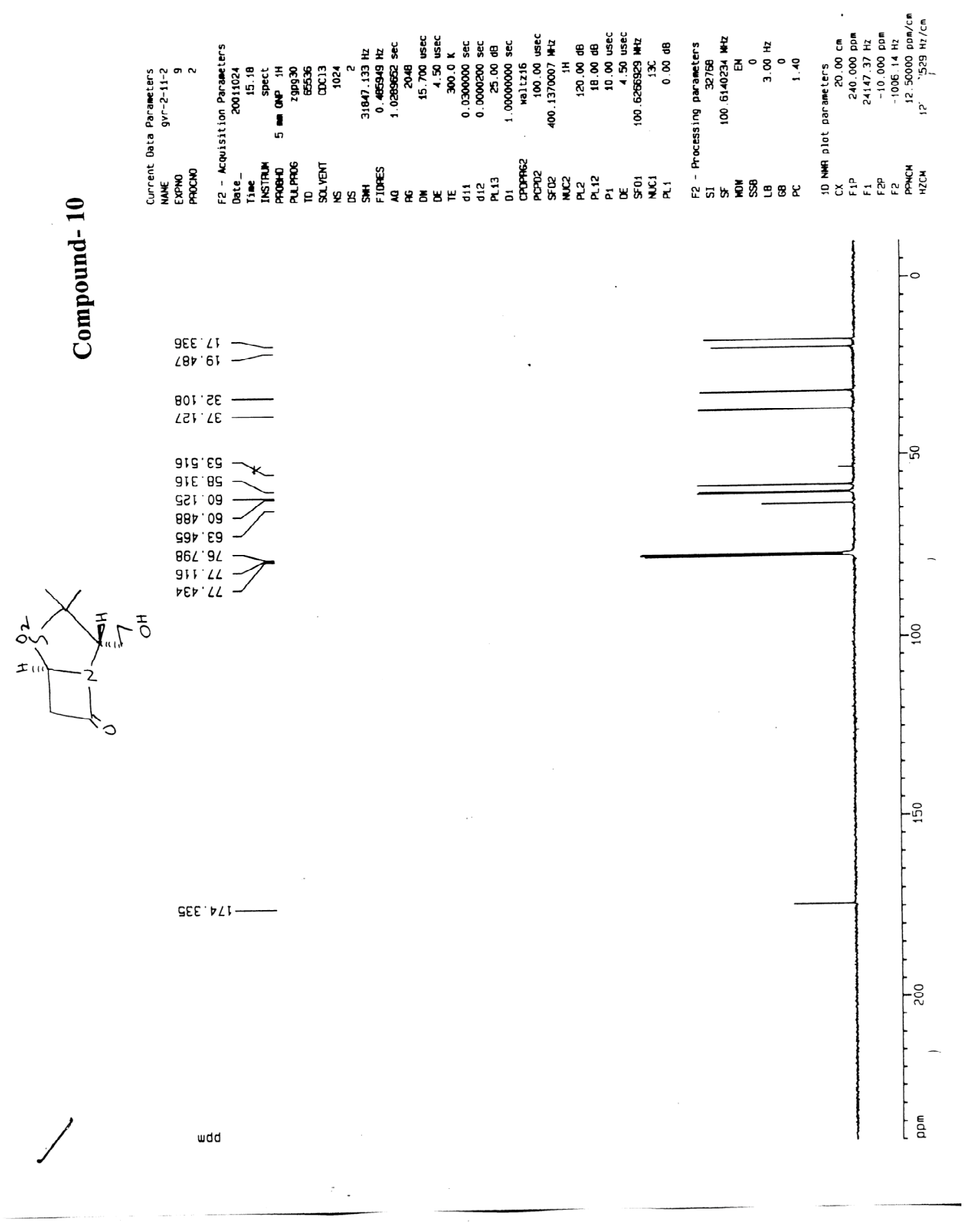




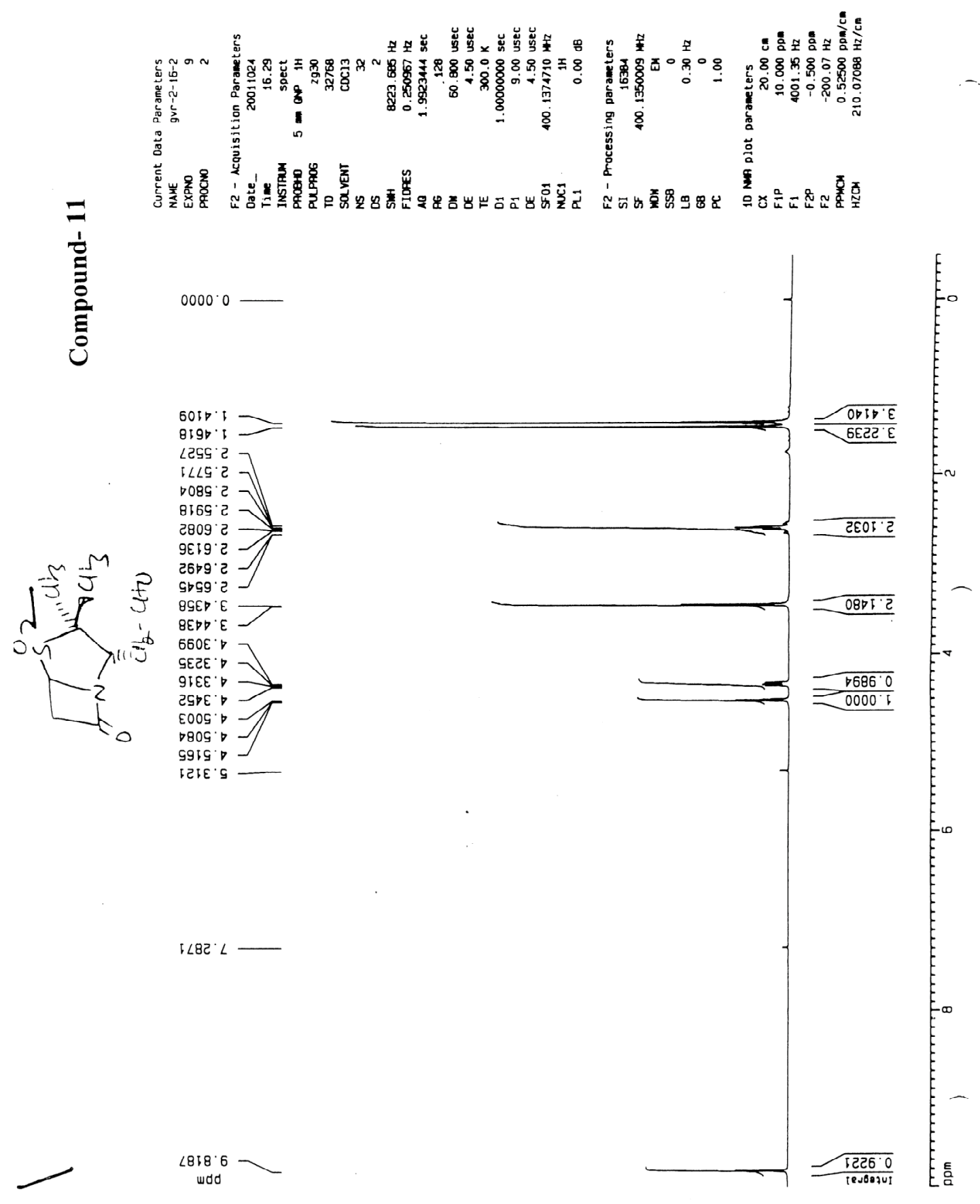



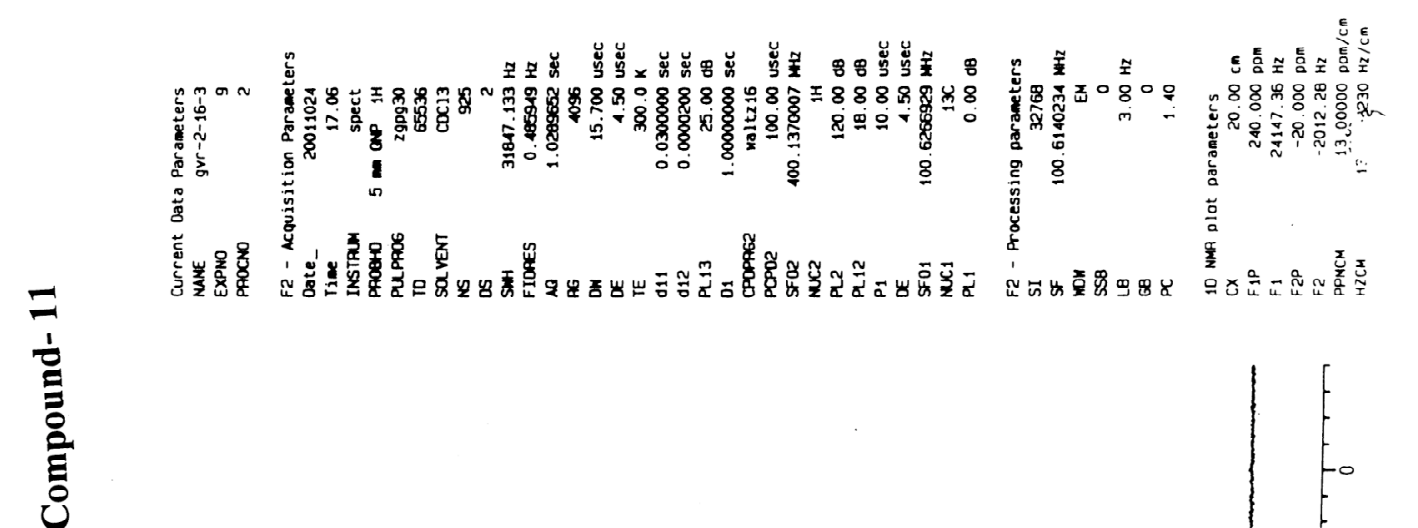

G25.81

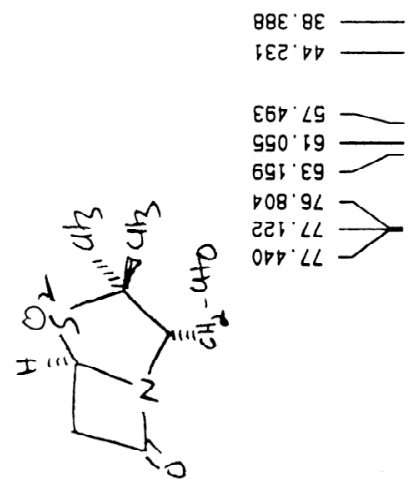

$988^{\circ} \cdot \angle \mathrm{T}$

$629 \cdot 865$

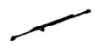

wdd

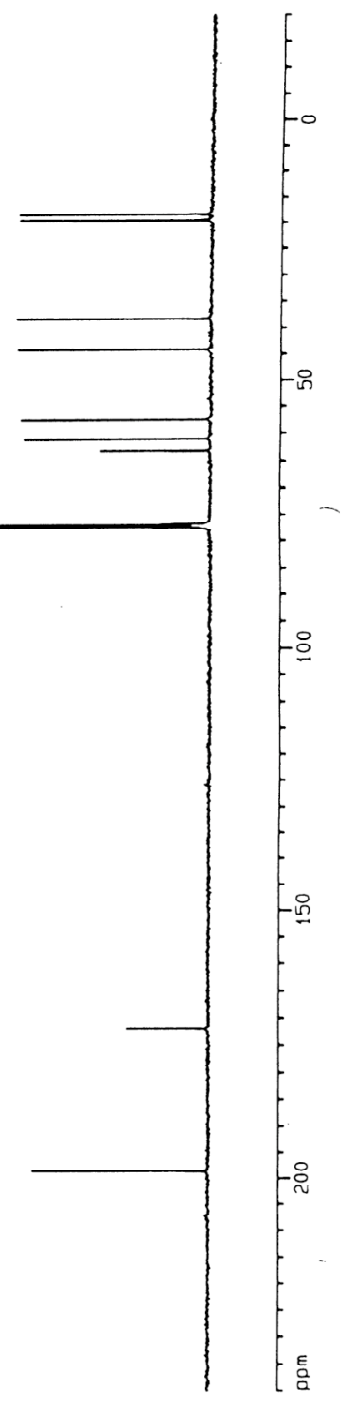




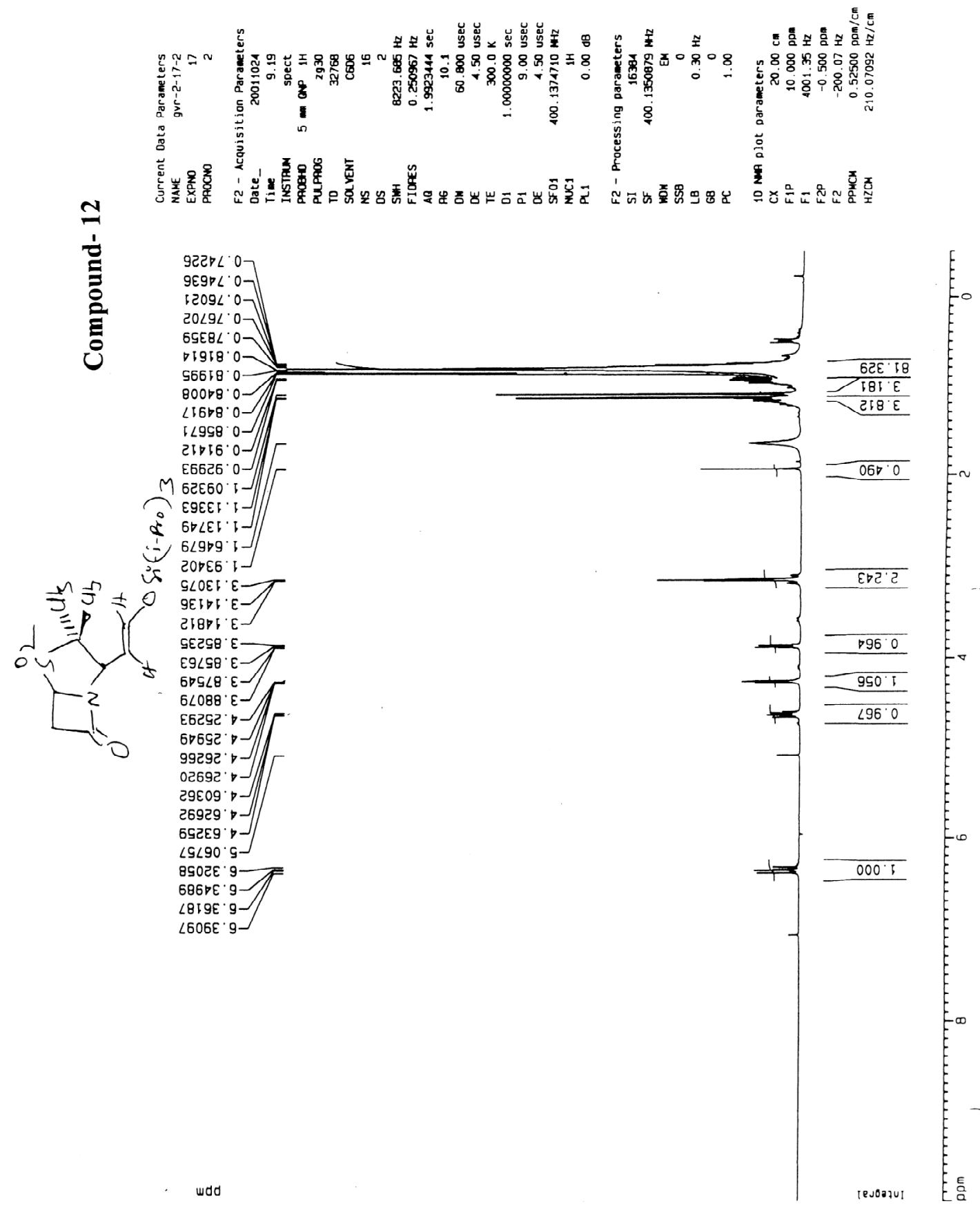




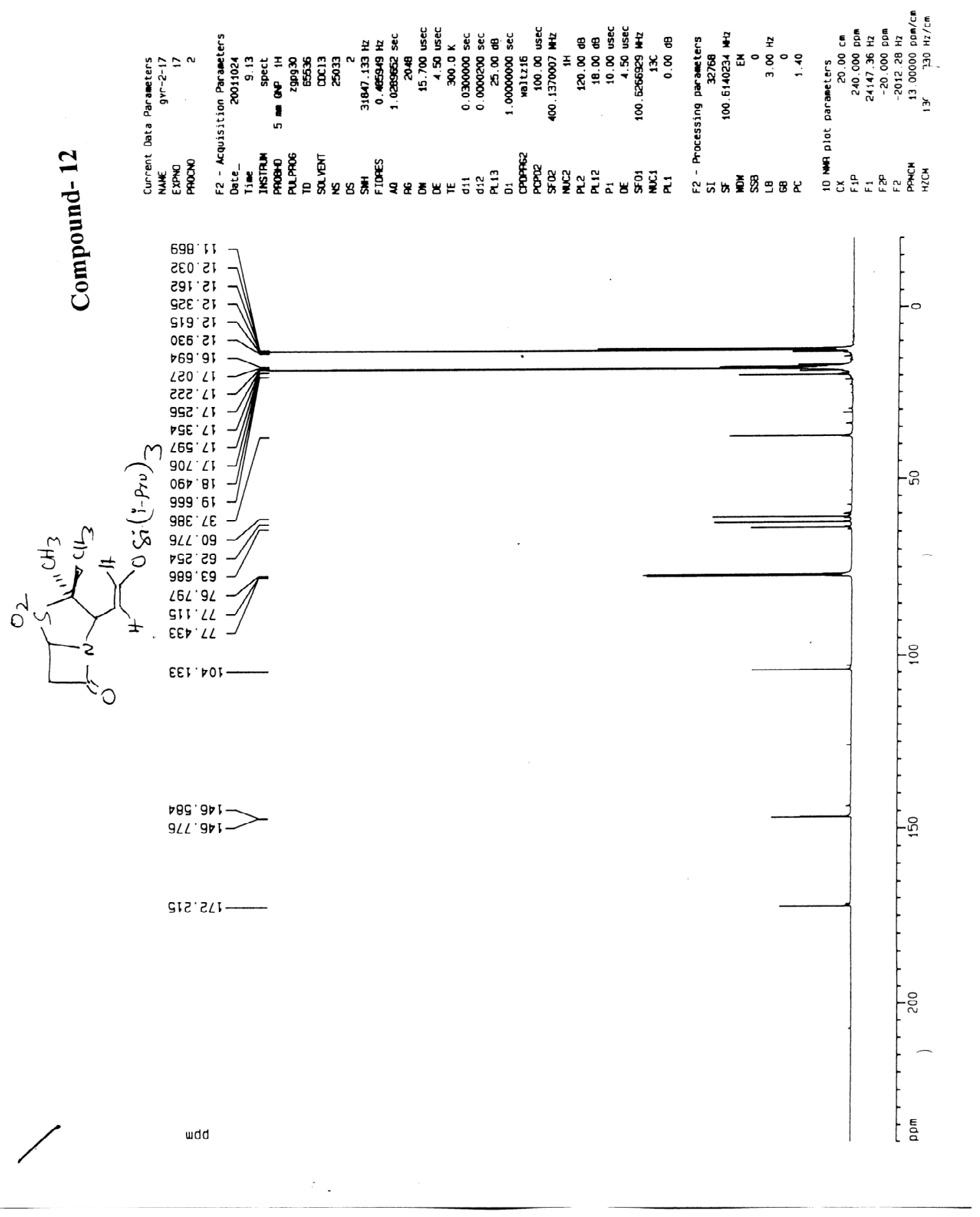




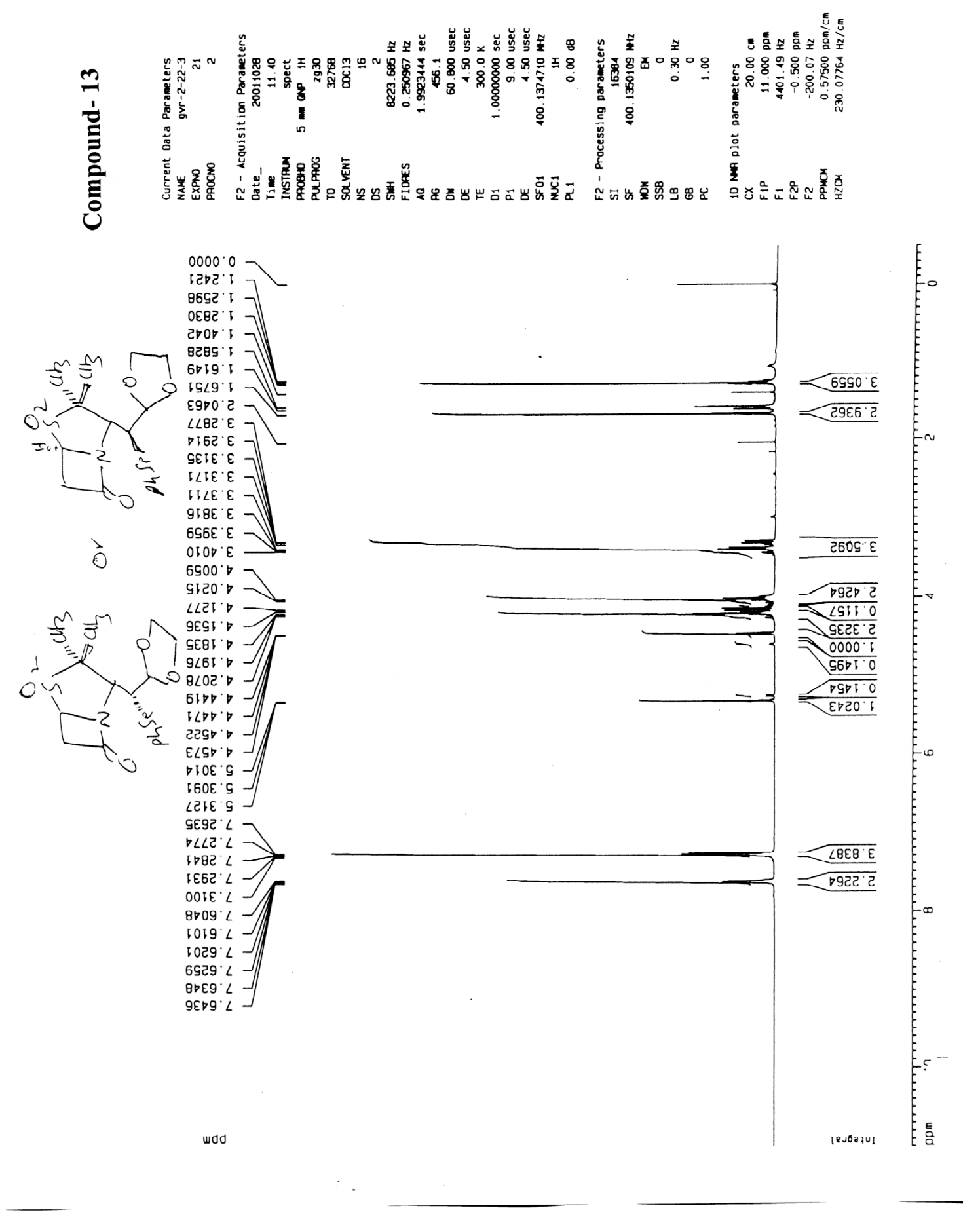




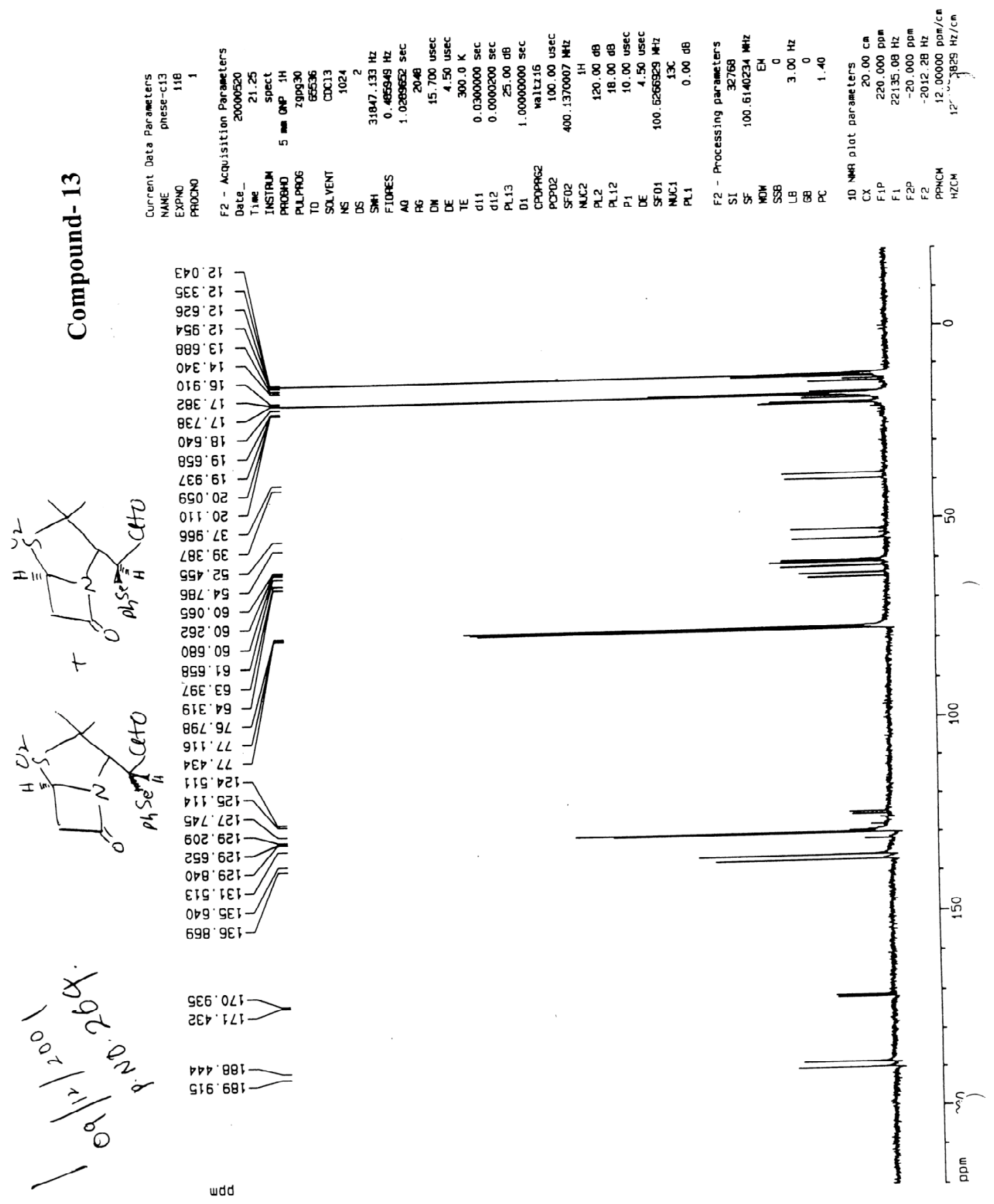




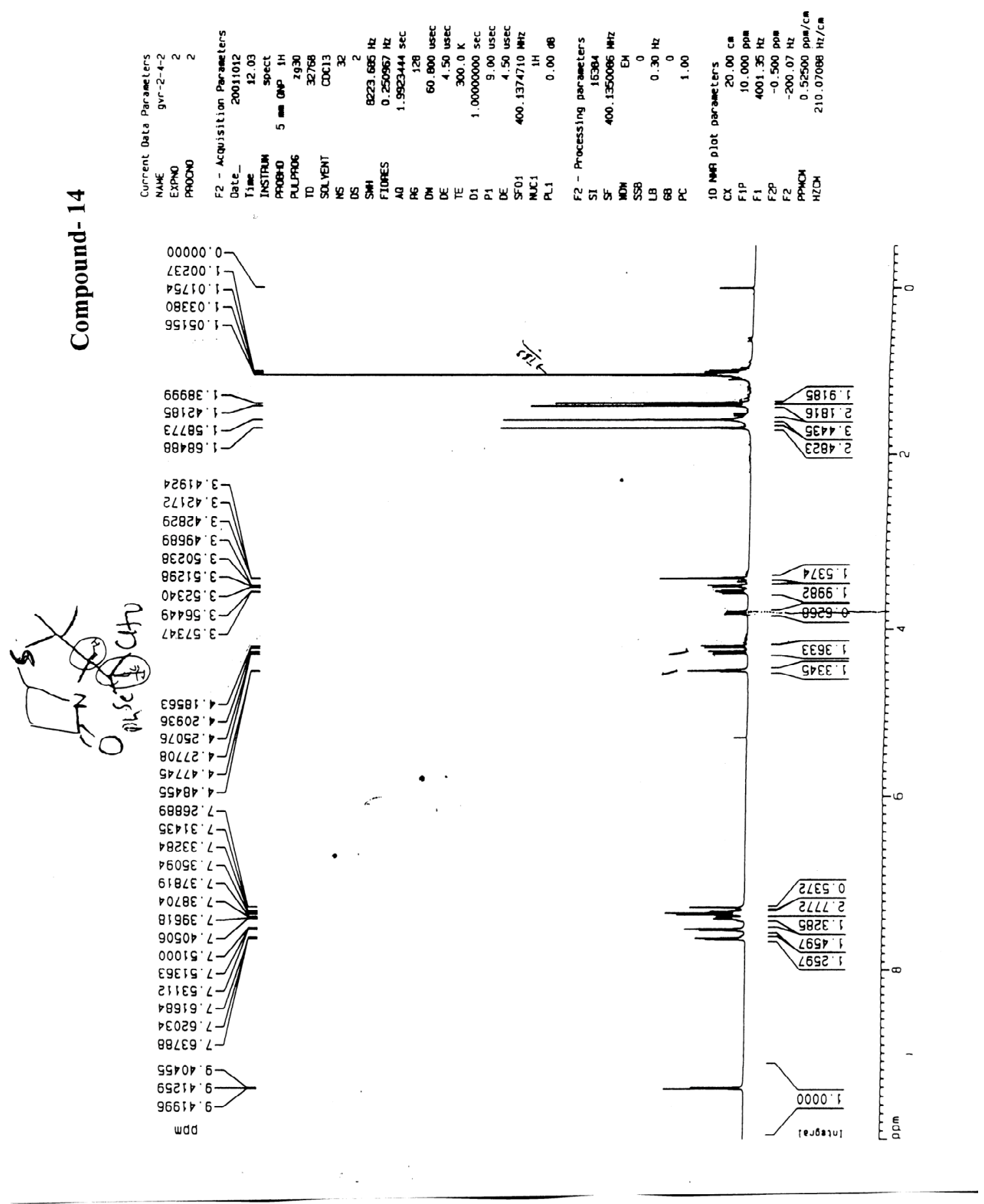




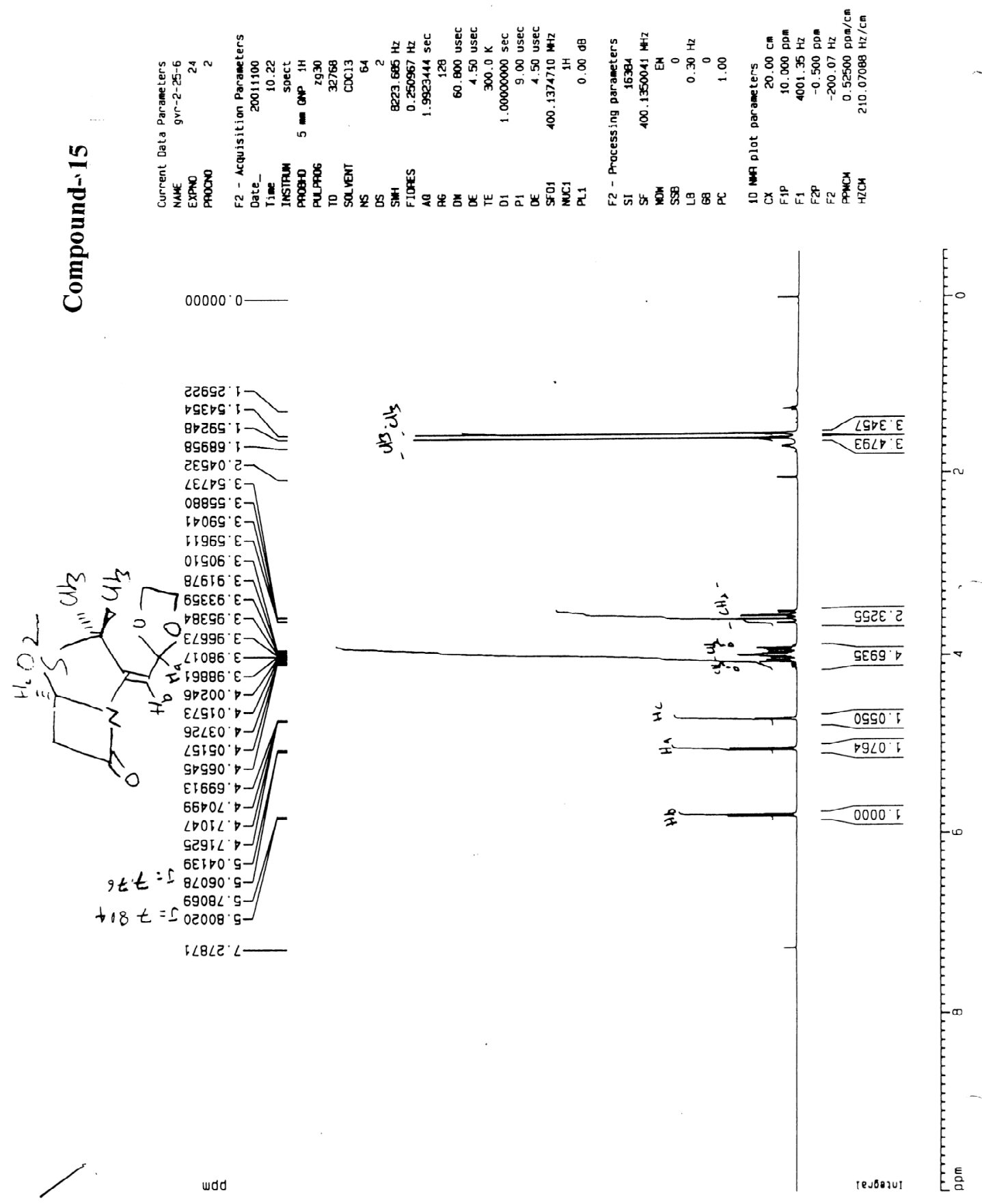




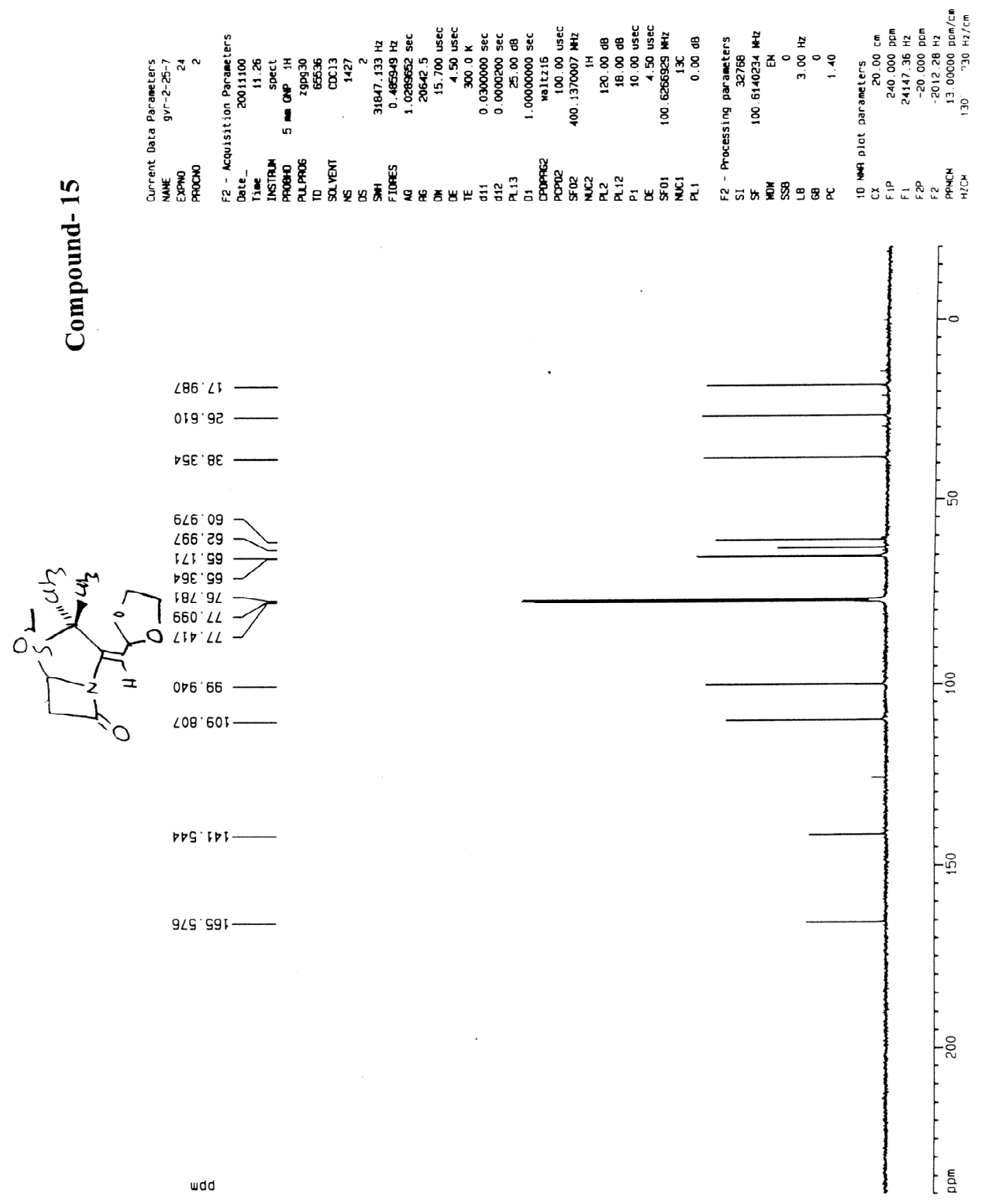




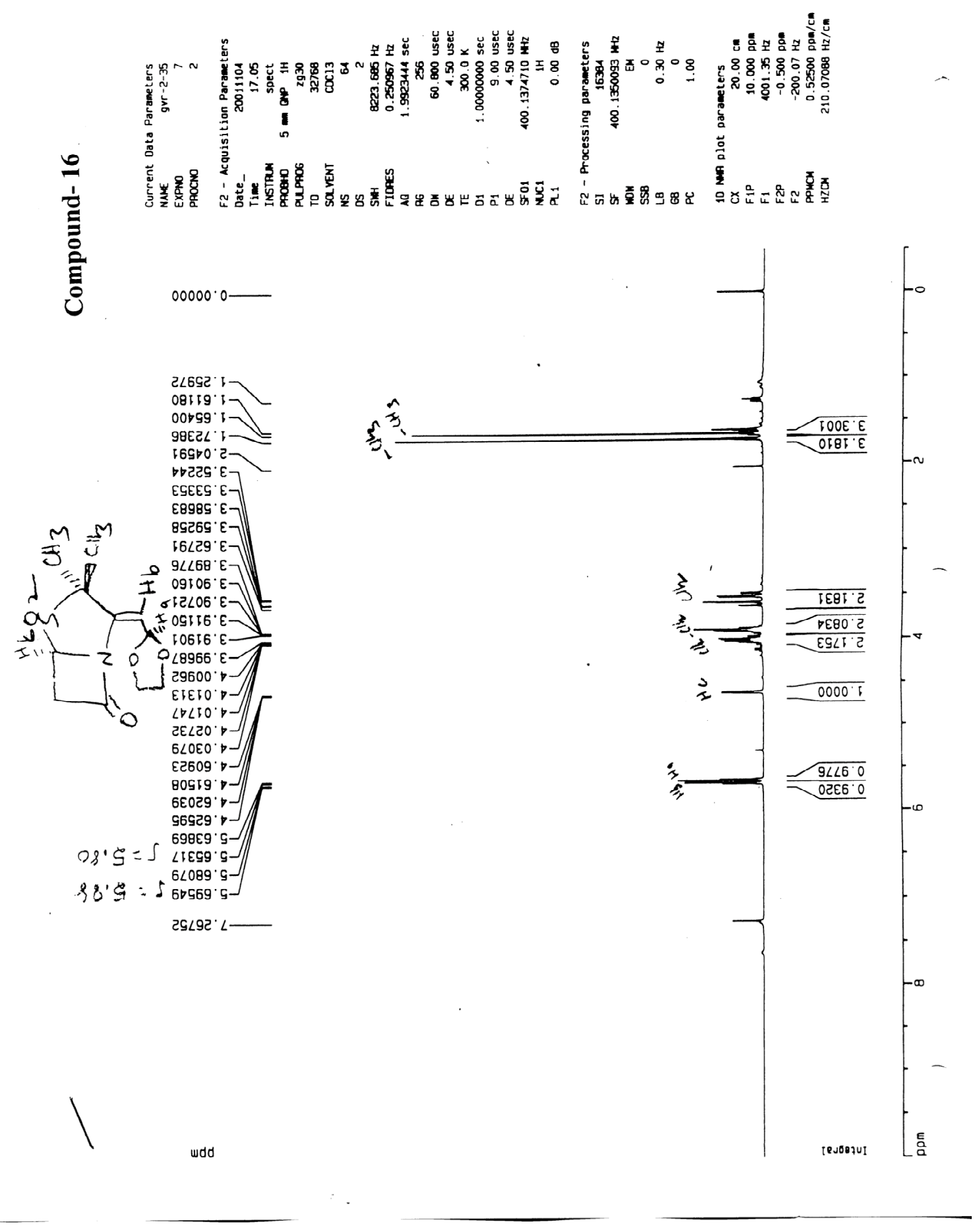




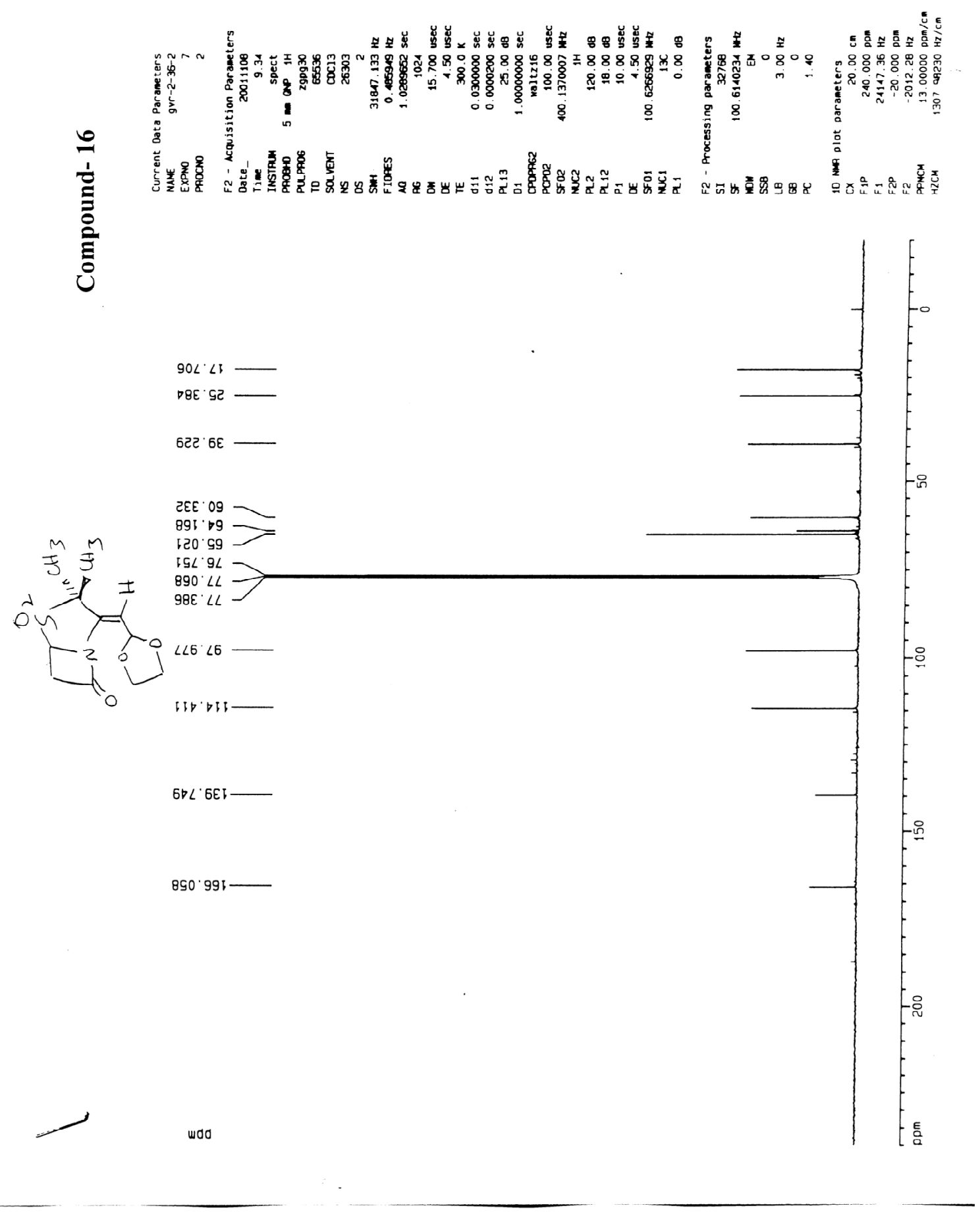




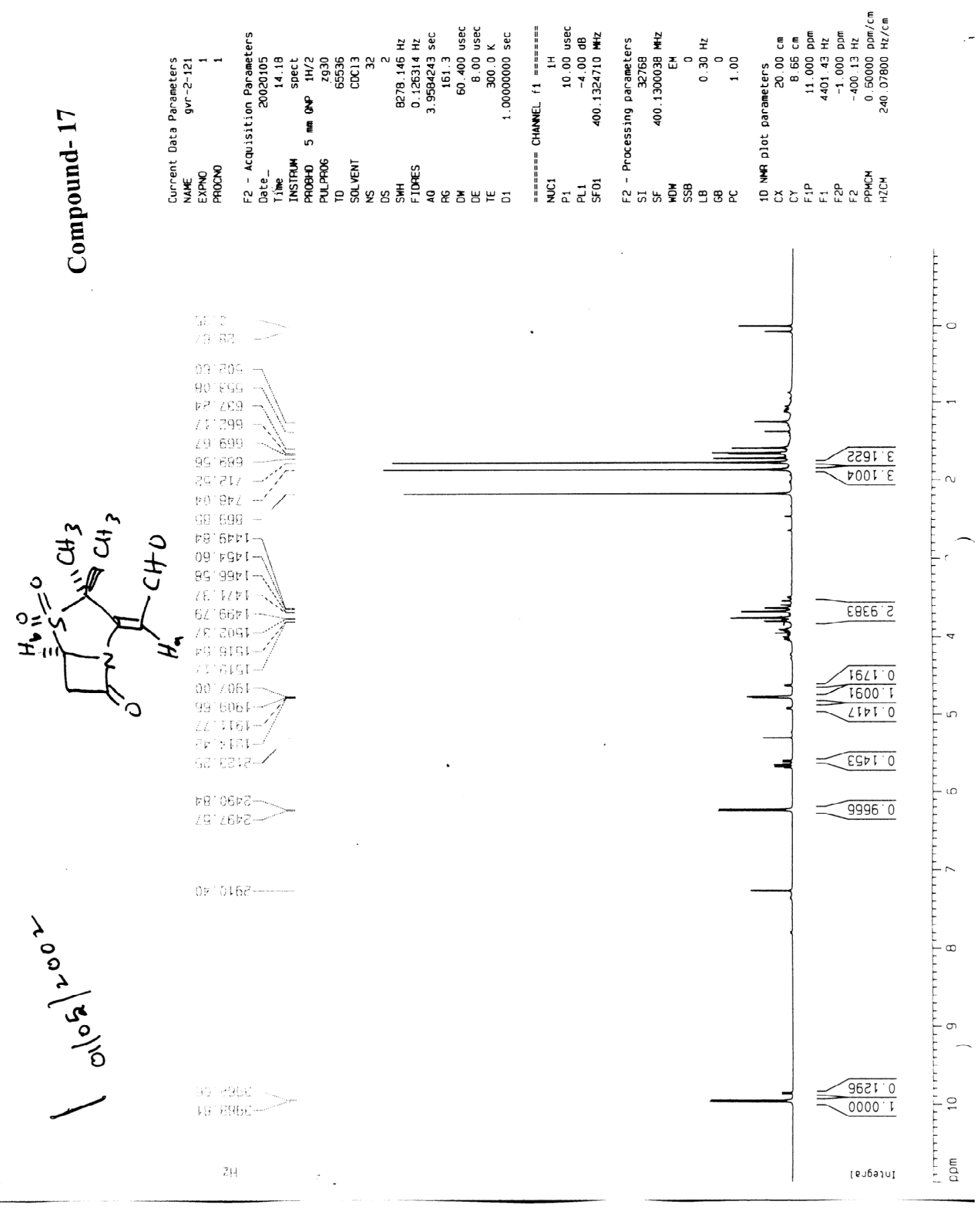




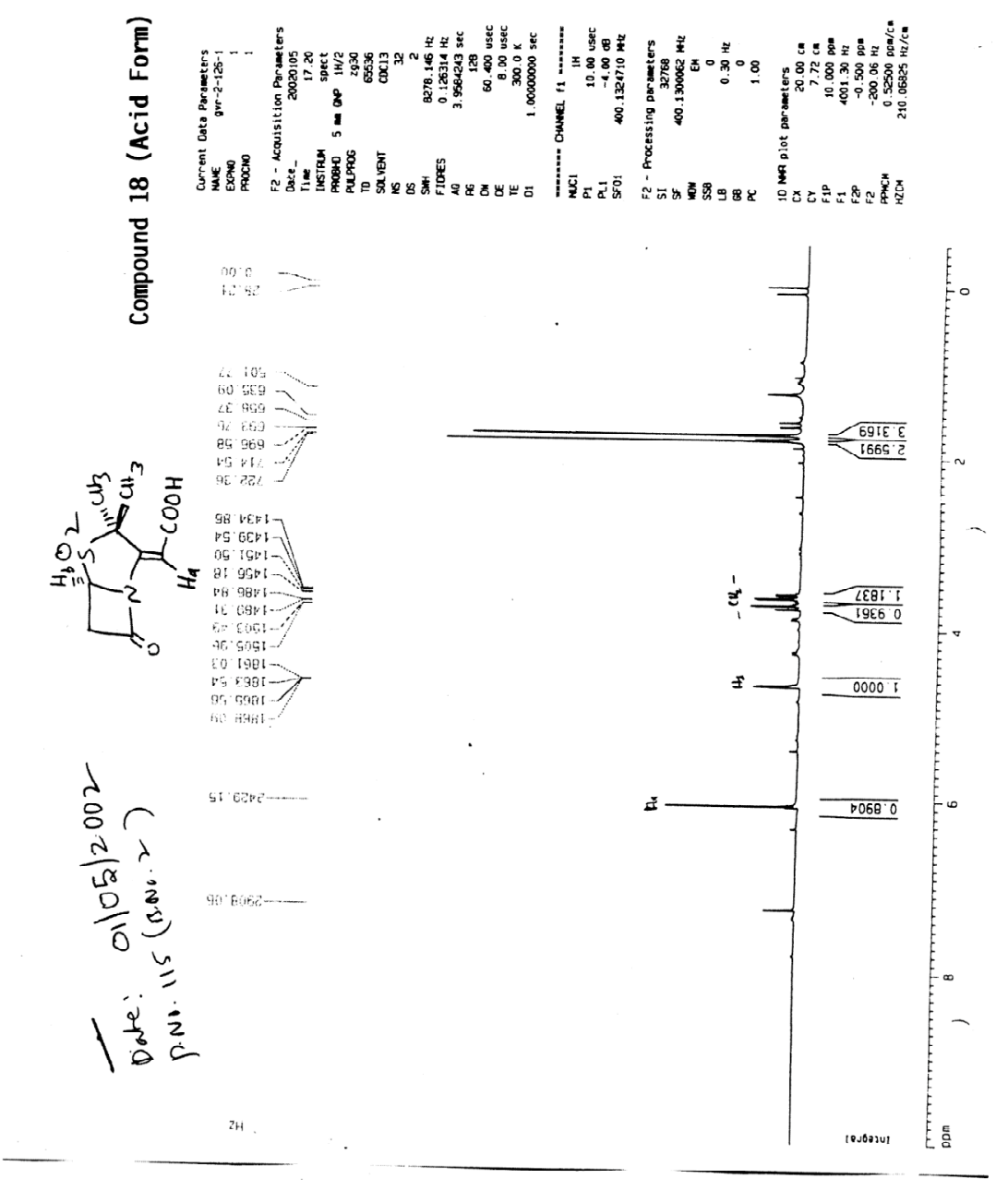




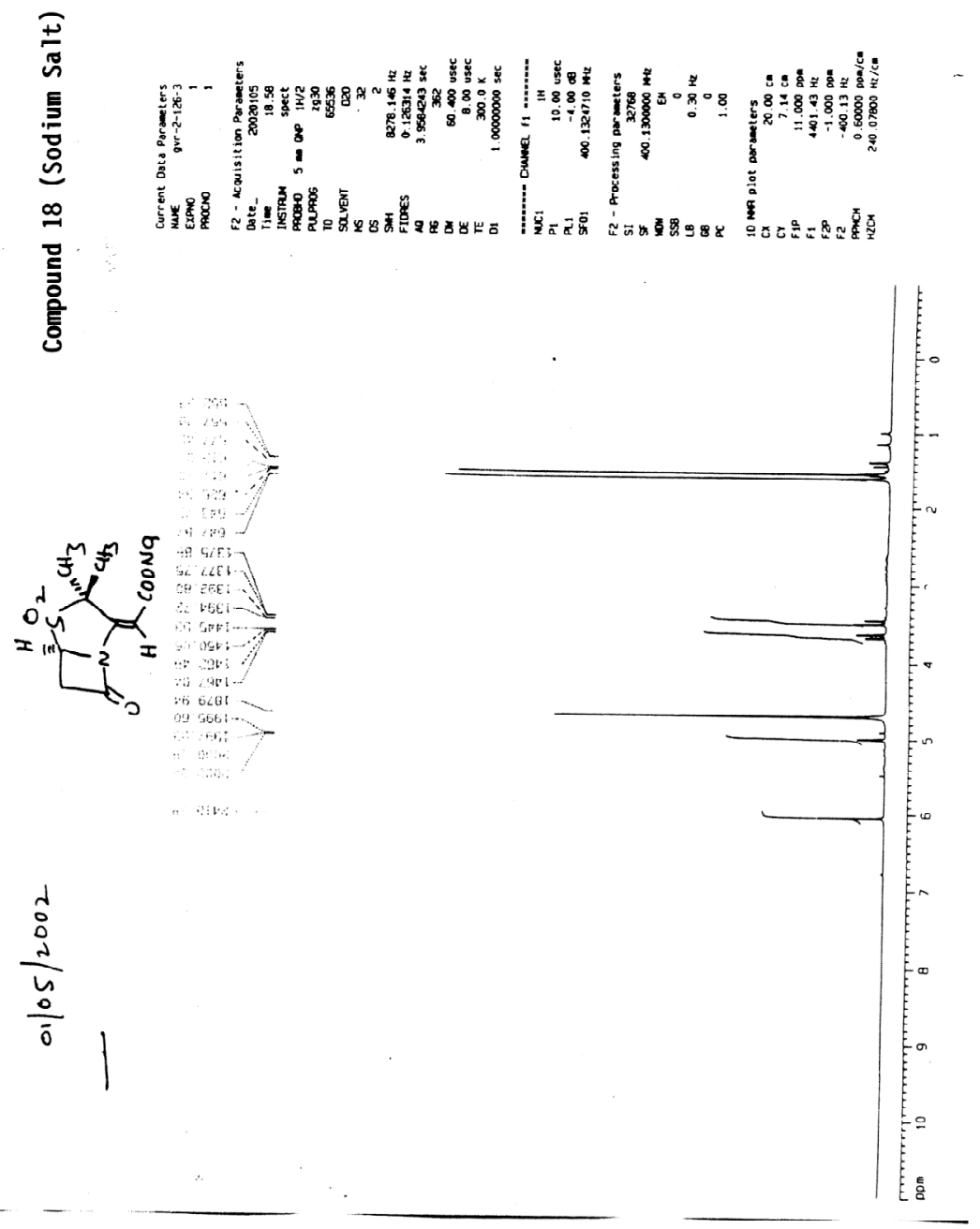




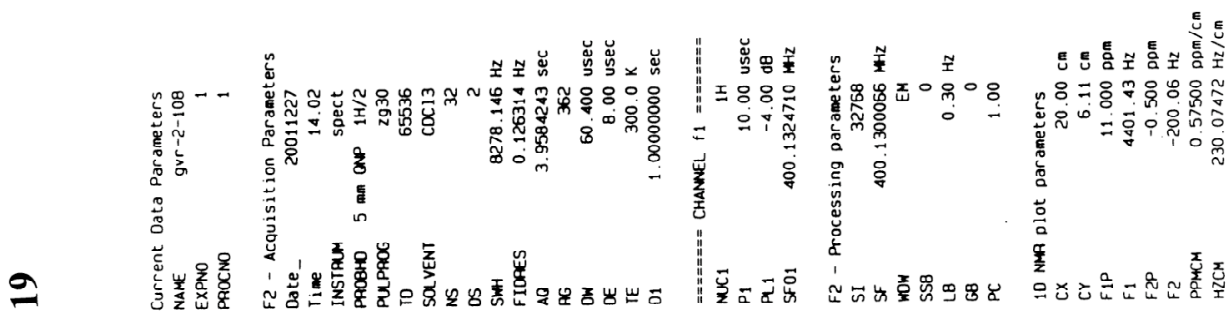

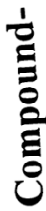
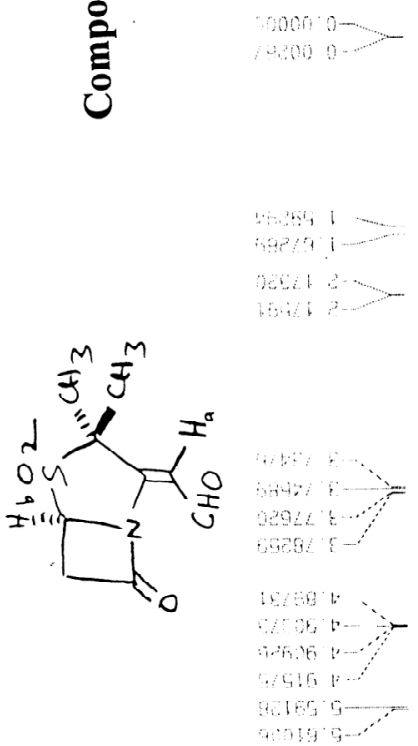

net. $\therefore$ on
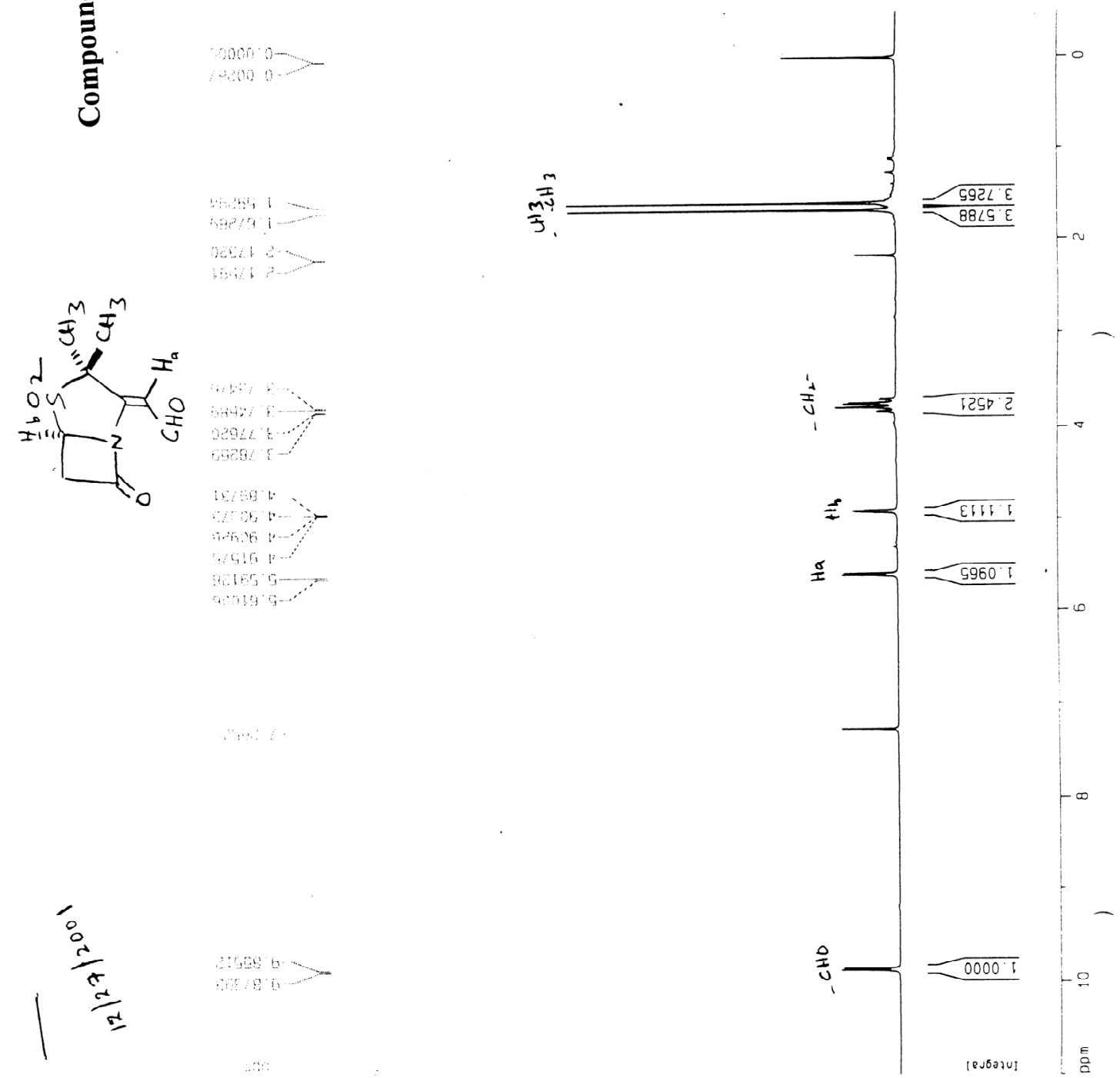


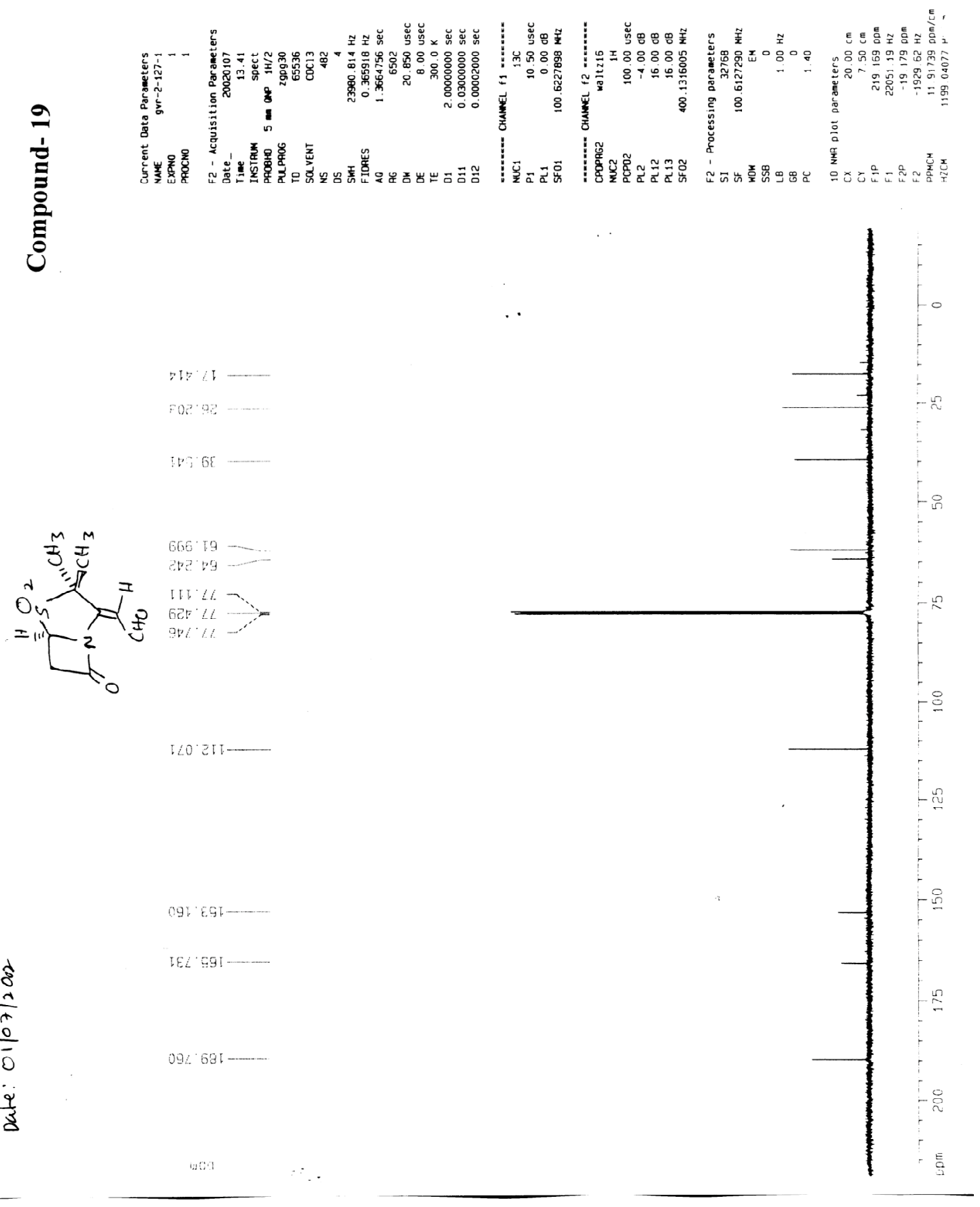




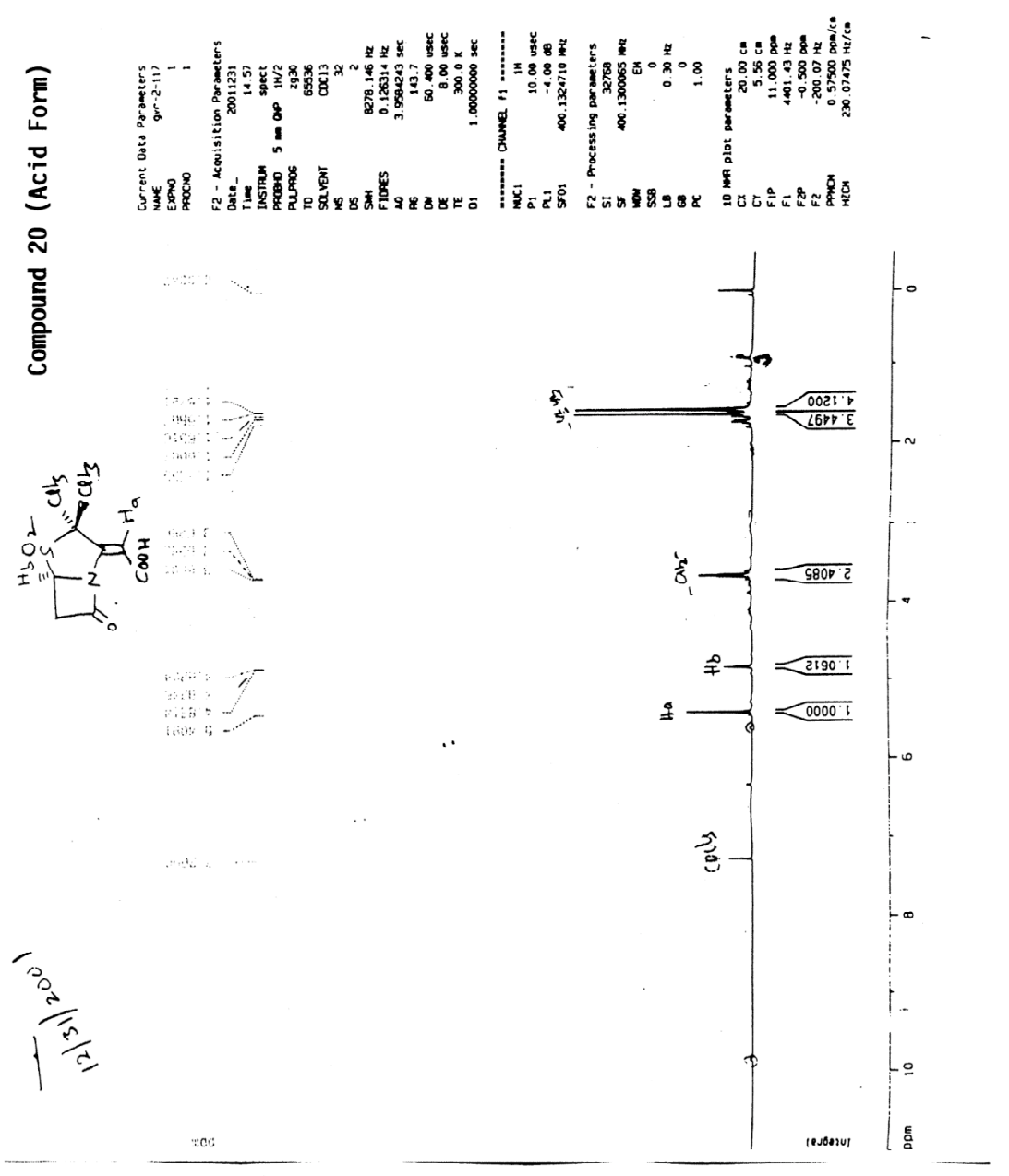




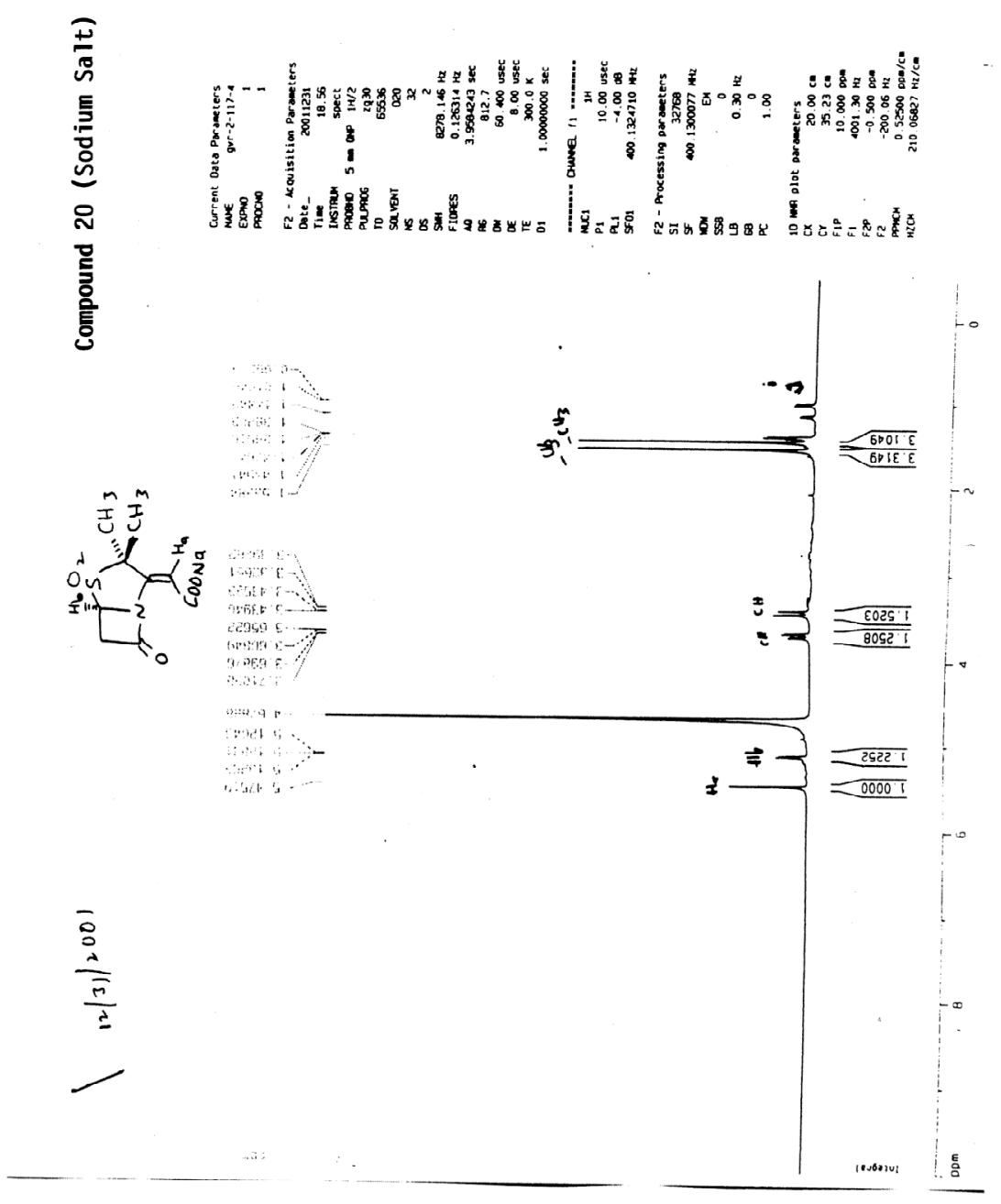


Table 1. Crystal data and structure refinement for compound 9.

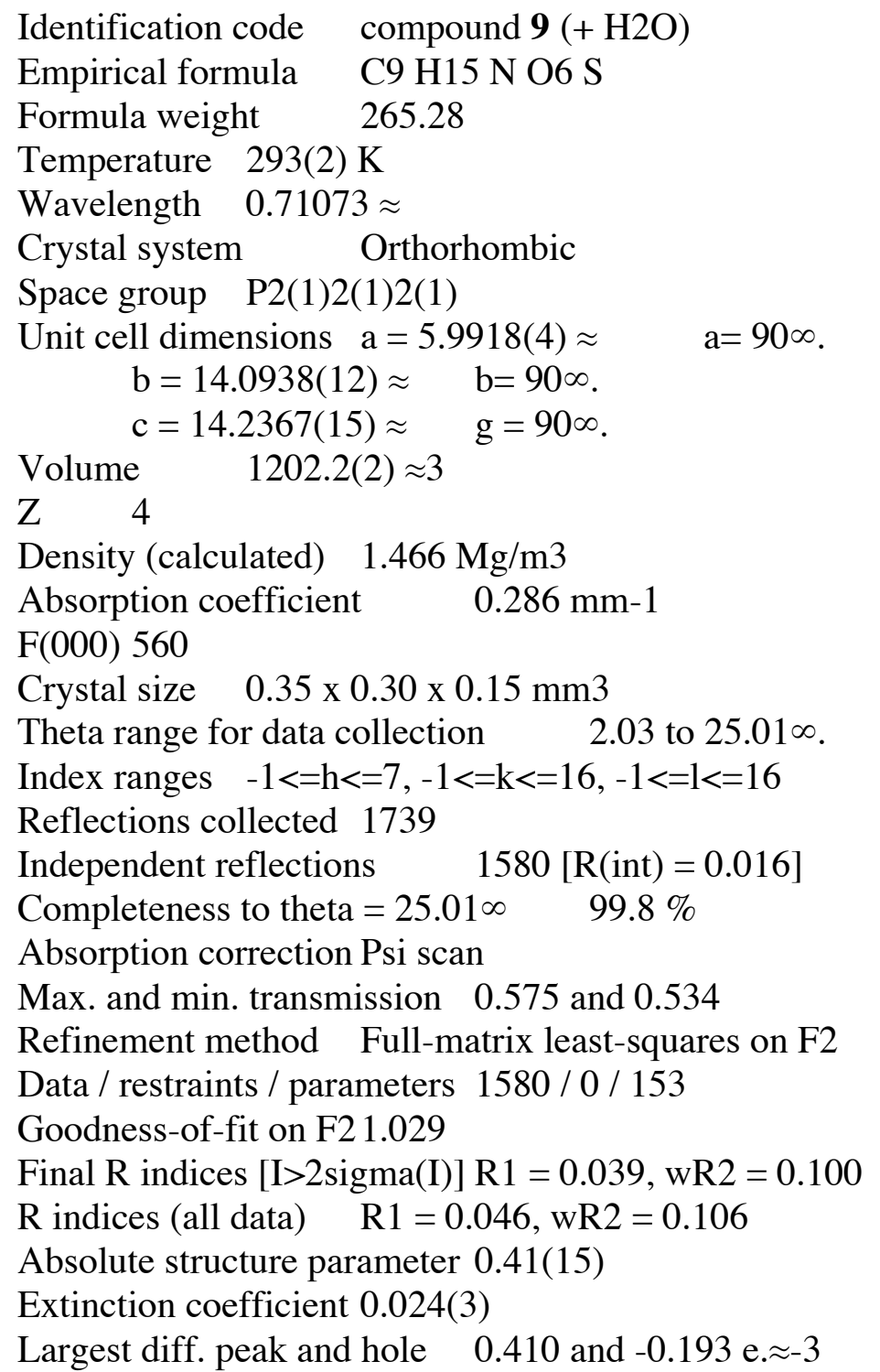


Table 2. Atomic coordinates ( x 104) and equivalent isotropic displacement parameters $(\approx 2 \mathrm{x}$ 103) for $9 . \mathrm{U}(\mathrm{eq})$ is defined as one third of the trace of the orthogonalized Uij tensor.

\begin{tabular}{|c|c|c|c|c|}
\hline \multirow[b]{2}{*}{$S(1)$} & \multirow{2}{*}{$\begin{array}{l}x \quad y \\
10951(2)\end{array}$} & \multirow{2}{*}{$\begin{array}{l}\mathrm{Z} \\
\mathrm{U}(21(1)\end{array}$} & \multicolumn{2}{|c|}{$\mathrm{U}(\mathrm{eq})$} \\
\hline & & & $3178(1)$ & $45(1)$ \\
\hline$C(2)$ & $9710(6)$ & $9056(2)$ & $2449(2)$ & $37(1)$ \\
\hline$C(3)$ & $8338(5)$ & $9616(2)$ & $3181(2)$ & $33(1)$ \\
\hline $\mathrm{N}(4)$ & $9443(5)$ & $9510(2)$ & $4102(2)$ & $37(1)$ \\
\hline$C(5)$ & $11394(6)$ & $8890(2)$ & $4168(2)$ & $44(1)$ \\
\hline$C(6)$ & $10536(8)$ & $8531(3)$ & $5120(3)$ & $60(1)$ \\
\hline$C(7)$ & $8456(8)$ & $9098(3)$ & $4898(2)$ & $49(1)$ \\
\hline $\mathrm{O}(8)$ & $6596(6)$ & $9186(3)$ & $5193(2)$ & $73(1)$ \\
\hline $\mathrm{O}(9)$ & $9226(5)$ & $7456(2)$ & $3417(2)$ & $59(1)$ \\
\hline $\mathrm{O}(10)$ & $13015(5)$ & $7770(2)$ & $2797(2)$ & $70(1)$ \\
\hline $\mathrm{C}(11)$ & $8257(7)$ & $8602(3)$ & $1702(3)$ & $54(1)$ \\
\hline$C(12)$ & 11598(6) & $9626(3)$ & 1999(3) & $51(1)$ \\
\hline$C(13)$ & $8012(7)$ & $10673(2)$ & $2935(2)$ & $42(1)$ \\
\hline$C(14)$ & $6634(6)$ & $11154(2)$ & $3661(2)$ & $39(1)$ \\
\hline $\mathrm{O}(15)$ & $7408(4)$ & $11662(2)$ & $4270(2)$ & $48(1)$ \\
\hline $\mathrm{O}(16)$ & $4505(4)$ & $10969(2)$ & $3597(2)$ & $56(1)$ \\
\hline $\mathrm{O}(17)$ & $6830(5)$ & $3434(2)$ & $5108(2)$ & $52(1)$ \\
\hline
\end{tabular}


Table 3. Bond lengths $[\approx]$ and angles [ $\infty]$ for 9.

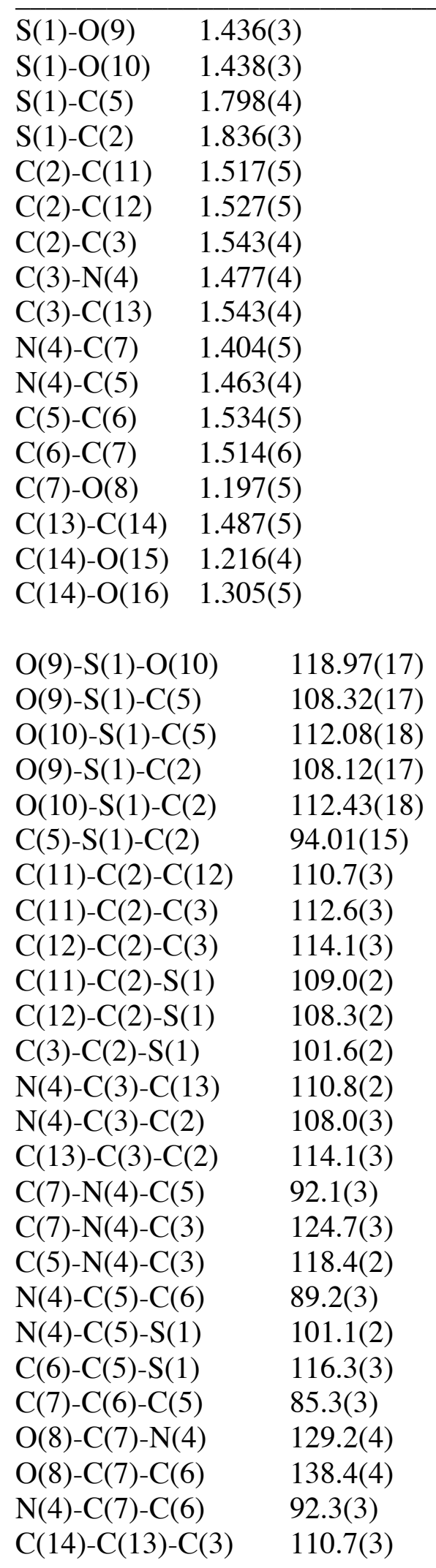


$\begin{array}{ll}\mathrm{O}(15)-\mathrm{C}(14)-\mathrm{O}(16) & 122.7(4) \\ \mathrm{O}(15)-\mathrm{C}(14)-\mathrm{C}(13) & 123.5(4) \\ \mathrm{O}(16)-\mathrm{C}(14)-\mathrm{C}(13) & 113.8(3)\end{array}$

Symmetry transformations used to generate equivalent atoms:

Table 4. Anisotropic displacement parameters $(\approx 2 \times 103)$ for 9 . The anisotropic displacement factor exponent takes the form: $-2 \mathrm{p} 2\left[\mathrm{~h} 2 \mathrm{a} * 2 \mathrm{U} 11+\ldots+2 \mathrm{~h} \mathrm{k} \mathrm{a}^{*} \mathrm{~b}^{*} \mathrm{U} 12\right.$ ]

U11 U22 U33 U23 U13 U12

$\begin{array}{lllllll}\overline{\mathrm{S}(1)} & 41(1) & 34(1) & 60(1) & -7(1) & 2(1) & 6(1) \\ \mathrm{C}(2) & 36(2) & 35(1) & 41(2) & -6(1) & 1(2) & -1(2) \\ \mathrm{C}(3) & 33(2) & 31(1) & 33(1) & -3(1) & 1(2) & 0(1) \\ \mathrm{N}(4) & 40(2) & 36(1) & 33(1) & -3(1) & 0(1) & 4(1) \\ \mathrm{C}(5) & 41(2) & 43(2) & 49(2) & -3(2) & -8(2) & 2(2) \\ \mathrm{C}(6) & 70(3) & 64(2) & 48(2) & 7(2) & -8(2) & 9(3) \\ \mathrm{C}(7) & 61(3) & 50(2) & 36(2) & 2(2) & -2(2) & 5(2) \\ \mathrm{O}(8) & 64(2) & 97(2) & 58(2) & 20(2) & 23(2) & 14(2) \\ \mathrm{O}(9) & 62(2) & 33(1) & 83(2) & 4(1) & -2(2) & -7(1) \\ \mathrm{O}(10) & 51(2) & 63(2) & 96(2) & -16(2) & 9(2) & 22(2) \\ \mathrm{C}(11) & 57(2) & 59(2) & 48(2) & -21(2) & -3(2) & -7(2) \\ \mathrm{C}(12) & 51(2) & 55(2) & 46(2) & -10(2) & 15(2) & -8(2) \\ \mathrm{C}(13) & 51(2) & 33(2) & 41(2) & 2(1) & 2(2) & 3(2) \\ \mathrm{C}(14) & 44(2) & 28(1) & 43(2) & 1(1) & -5(2) & 6(2) \\ \mathrm{O}(15) & 42(1) & 48(1) & 54(1) & -18(1) & -6(1) & 0(1) \\ \mathrm{O}(16) & 38(1) & 58(2) & 73(2) & -28(1) & -4(1) & 1(1)\end{array}$


Table 5. Hydrogen coordinates ( $\mathrm{x}$ 104) and isotropic displacement parameters $(\approx 2 \mathrm{x} 103)$ for 9.

\begin{tabular}{|c|c|c|c|c|c|}
\hline & $\mathrm{x}$ & $\mathrm{y}$ & $\mathrm{z}$ & $\mathrm{U}(\mathrm{eq})$ & \\
\hline $\mathrm{H}(3)$ & 6859 & 9322 & 3223 & 39 & \\
\hline $\mathrm{H}(5)$ & 12841 & 9214 & 4184 & 53 & \\
\hline $\mathrm{H}(6 \mathrm{~A})$ & 10304 & 7851 & 5150 & 72 & \\
\hline $\mathrm{H}(6 \mathrm{~B})$ & 11372 & 8760 & 5657 & 72 & \\
\hline $\mathrm{H}(11 \mathrm{~A})$ & & 7590 & 9087 & 1321 & 82 \\
\hline $\mathrm{H}(11 \mathrm{~B})$ & & 7106 & 8234 & 1997 & 82 \\
\hline $\mathrm{H}(11 \mathrm{C})$ & & 9155 & 8196 & 1314 & 82 \\
\hline $\mathrm{H}(12 \mathrm{~A})$ & & 12571 & 9204 & 1664 & 76 \\
\hline $\mathrm{H}(12 \mathrm{~B})$ & & 12427 & 9948 & 2480 & 76 \\
\hline $\mathrm{H}(12 \mathrm{C})$ & & 10982 & 10083 & 1572 & 76 \\
\hline $\mathrm{H}(13 \mathrm{~A})$ & & 9456 & 10981 & 2893 & 50 \\
\hline $\mathrm{H}(13 \mathrm{~B})$ & & 7286 & 10727 & 2328 & 50 \\
\hline $\mathrm{H}(16)$ & 3837 & 11238 & 4023 & 84 & \\
\hline $\mathrm{H}(17 \mathrm{~A})$ & & 7056 & 2760 & 4781 & 78 \\
\hline $\mathrm{H}(17 \mathrm{~B})$ & & 5036 & 3521 & 5212 & 78 \\
\hline
\end{tabular}


Table 6. Crystal data and structure refinement for compound $\mathbf{1 6 .}$

$\begin{array}{ll}\text { Identification code } & \text { compound } 16 \\ \text { Empirical formula } & \text { C11 H15 N O5 S } \\ \text { Formula weight } & 273.30 \\ \text { Temperature } 293(2) \mathrm{K} \\ \text { Wavelength } 0.71073 \approx \\ \text { Crystal system } \quad \text { Orthorhombic }\end{array}$

Space group P2(1)2(1)2(1)

Unit cell dimensions $\mathrm{a}=8.2835(3) \approx \quad \mathrm{a}=90 \infty$.

$$
\begin{array}{ll}
\mathrm{b}=8.7673(5) \approx & \mathrm{b}=90 \infty . \\
\mathrm{c}=17.6458(10) \approx & \mathrm{g}=90 \infty .
\end{array}
$$

Volume $\quad 1281.5(1) \approx 3$

Z 4

Density (calculated) $1.417 \mathrm{Mg} / \mathrm{m} 3$

Absorption coefficient $\quad 0.265 \mathrm{~mm}-1$

$\mathrm{F}(000) 576$

Crystal size $\quad 0.40 \times 0.35 \times 0.20 \mathrm{~mm} 3$

Theta range for data collection 2.31 to $24.99 \infty$.

Index ranges $-1<=\mathrm{h}<=9,-1<=\mathrm{k}<=10,-1<=1<=20$

Reflections collected 1798

Independent reflections $\quad 1650[\mathrm{R}(\mathrm{int})=0.015]$

Completeness to theta $=24.99 \infty \quad 99.3 \%$

Absorption correction Psi scan

Max. and min. transmission 0.449 and 0.434

Refinement method Full-matrix least-squares on F2

Data / restraints / parameters 1650 / 0 / 166

Goodness-of-fit on F2 1.069

Final $\mathrm{R}$ indices $[\mathrm{I}>2 \operatorname{sigma}(\mathrm{I})] \mathrm{R} 1=0.031, \mathrm{wR} 2=0.081$

$\mathrm{R}$ indices (all data) $\mathrm{R} 1=0.035, \mathrm{wR} 2=0.084$

Absolute structure parameter 0.06(11)

Extinction coefficient 0.024(3)

Largest diff. peak and hole 0.180 and -0.189 e. $\approx-3$ 
Table 7. Atomic coordinates (x 104) and equivalent isotropic displacement parameters $(\approx 2 \mathrm{x} 103)$ for 16 . $\mathrm{U}(\mathrm{eq})$ is defined as one third of the trace of the orthogonalized Uij tensor.

\begin{tabular}{|c|c|c|c|c|}
\hline & $\mathrm{x}$ & $\mathrm{z}$ & & \\
\hline $\mathrm{S}(1)$ & 8494(1) & $2955(1)$ & $4068(1)$ & $45(1)$ \\
\hline$C(2)$ & $6897(3)$ & $2684(3)$ & $3362(1)$ & $40(1)$ \\
\hline $\mathrm{C}(3)$ & 7861(3) & $2468(3)$ & 2637(1) & $38(1)$ \\
\hline $\mathrm{N}(4)$ & $9440(2)$ & $1920(3)$ & $2807(1)$ & $38(1)$ \\
\hline$C(5)$ & $9830(3)$ & $1640(3)$ & $3608(1)$ & $44(1)$ \\
\hline$C(6)$ & $11564(3)$ & 2192(4) & $3463(2)$ & $57(1)$ \\
\hline$C(7)$ & $10964(3)$ & $2558(3)$ & $2661(2)$ & $47(1)$ \\
\hline $\mathrm{O}(8)$ & $11483(3)$ & $3167(3)$ & $2105(1)$ & $68(1)$ \\
\hline $\mathrm{O}(9)$ & $9139(3)$ & $4459(2)$ & $3975(1)$ & $63(1)$ \\
\hline $\mathrm{O}(10)$ & $8006(3)$ & $2460(3)$ & $4806(1)$ & $68(1)$ \\
\hline $\mathrm{C}(11)$ & $5797(4)$ & $4072(4)$ & $3364(2)$ & $60(1)$ \\
\hline$C(12)$ & $5969(4)$ & $1233(4)$ & $3551(2)$ & $52(1)$ \\
\hline$C(13)$ & $7310(3)$ & $2697(3)$ & 1944(1) & $46(1)$ \\
\hline$C(14)$ & $8153(3)$ & $2267(3)$ & $1235(1)$ & $45(1)$ \\
\hline $\mathrm{O}(15)$ & $8392(3)$ & $3543(2)$ & $761(1) 72(1)$ & \\
\hline$C(16)$ & $8098(5)$ & $3059(5)$ & $7(2) \quad 80(1)$ & \\
\hline$C(17)$ & $7671(5)$ & $1437(5)$ & $63(2) \quad 76(1)$ & \\
\hline $\mathrm{O}(18)$ & $7171(3)$ & $1256(2)$ & $827(1) 62(1)$ & \\
\hline
\end{tabular}




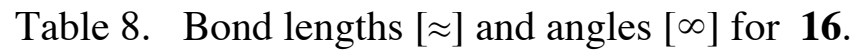

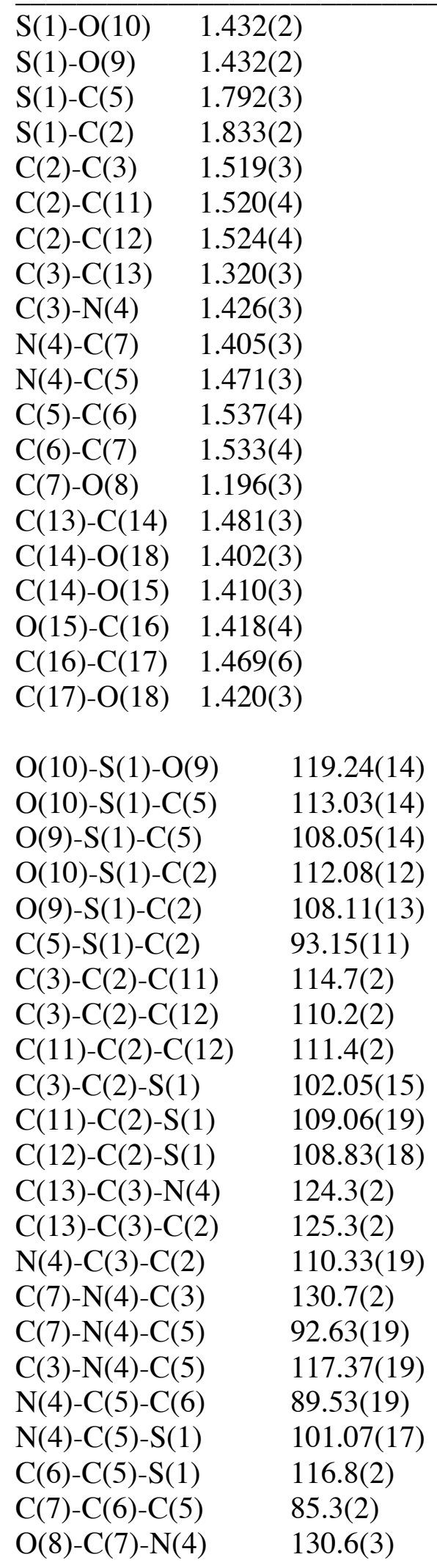




$\begin{array}{ll}\mathrm{O}(8)-\mathrm{C}(7)-\mathrm{C}(6) & 137.2(3) \\ \mathrm{N}(4)-\mathrm{C}(7)-\mathrm{C}(6) & 92.20(19) \\ \mathrm{C}(3)-\mathrm{C}(13)-\mathrm{C}(14) & 125.5(2) \\ \mathrm{O}(18)-\mathrm{C}(14)-\mathrm{O}(15) & 106.1(2) \\ \mathrm{O}(18)-\mathrm{C}(14)-\mathrm{C}(13) & 108.7(2) \\ \mathrm{O}(15)-\mathrm{C}(14)-\mathrm{C}(13) & 111.4(2) \\ \mathrm{C}(14)-\mathrm{O}(15)-\mathrm{C}(16) & 107.2(2) \\ \mathrm{O}(15)-\mathrm{C}(16)-\mathrm{C}(17) & 105.6(2) \\ \mathrm{O}(18)-\mathrm{C}(17)-\mathrm{C}(16) & 104.0(3) \\ \mathrm{C}(14)-\mathrm{O}(18)-\mathrm{C}(17) & 104.4(2)\end{array}$

Symmetry transformations used to generate equivalent atoms: 
Table 9. Anisotropic displacement parameters $(\approx 2 \times 103)$ for 16 . The anisotropic displacement factor exponent takes the form: $-2 \mathrm{p} 2\left[\mathrm{~h} 2 \mathrm{a} * 2 \mathrm{U} 11+\ldots+2 \mathrm{~h} \mathrm{k} \mathrm{a}^{*} \mathrm{~b}^{*} \mathrm{U} 12\right.$ ]

\section{U11 U22 U33 U23 U13 U12}

$\begin{array}{lllllll}\overline{\mathrm{S}(1)} & 45(1) & 52(1) & 40(1) & -7(1) & 1(1) & -3(1) \\ \mathrm{C}(2) & 35(1) & 46(1) & 39(1) & -5(1) & 1(1) & 4(1) \\ \mathrm{C}(3) & 36(1) & 36(1) & 41(1) & 0(1) & 1(1) & 1(1) \\ \mathrm{N}(4) & 35(1) & 41(1) & 38(1) & 2(1) & 2(1) & 4(1) \\ \mathrm{C}(5) & 40(1) & 51(2) & 42(1) & 6(1) & -3(1) & 4(1) \\ \mathrm{C}(6) & 37(1) & 77(2) & 58(2) & 6(2) & -2(1) & 6(2) \\ \mathrm{C}(7) & 39(1) & 51(2) & 53(2) & -2(1) & 7(1) & 1(1) \\ \mathrm{O}(8) & 60(1) & 83(2) & 63(1) & 13(1) & 14(1) & -17(1) \\ \mathrm{O}(9) & 61(1) & 52(1) & 77(1) & -18(1) & 2(1) & -10(1) \\ \mathrm{O}(10) & 70(1) & 98(2) & 36(1) & -3(1) & 5(1) & -4(2) \\ \mathrm{C}(11) & 58(2) & 63(2) & 58(2) & -6(2) & 4(2) & 23(2) \\ \mathrm{C}(12) & 41(2) & 64(2) & 52(2) & -3(1) & 3(1) & -9(2) \\ \mathrm{C}(13) & 44(1) & 50(2) & 44(1) & -1(1) & -4(1) & 9(1) \\ \mathrm{C}(14) & 57(2) & 42(1) & 38(1) & 0(1) & -3(1) & -2(1) \\ \mathrm{O}(15) & 115(2) & 47(1) & 52(1) & 7(1) & 8(1) & -14(2) \\ \mathrm{C}(16) & 94(3) & 99(3) & 47(2) & 22(2) & -5(2) & 0(3) \\ \mathrm{C}(17) & 88(3) & 95(3) & 43(2) & -17(2) & -4(2) & -7(3) \\ \mathrm{O}(18) & 77(1) & 59(1) & 50(1) & -9(1) & 2(1) & -20(1)\end{array}$


Table 10. Hydrogen coordinates ( $\mathrm{x}$ 104) and isotropic displacement parameters $(\approx 2 \mathrm{x} 103)$ for 16.

\begin{tabular}{|c|c|c|c|c|c|}
\hline & $\mathrm{X}$ & $\mathrm{y}$ & $\mathrm{z}$ & $\mathrm{U}(\mathrm{eq})$ & \\
\hline $\mathrm{H}(5)$ & 9732 & 577 & 3774 & 53 & \\
\hline $\mathrm{H}(6 \mathrm{~A})$ & 11878 & 3078 & 3757 & 69 & \\
\hline $\mathrm{H}(6 \mathrm{~B})$ & 12375 & 1396 & 3486 & 69 & \\
\hline $\mathrm{H}(11 \mathrm{~A})$ & & 5296 & 4169 & 3853 & 89 \\
\hline $\mathrm{H}(11 \mathrm{~B})$ & & 4980 & 3953 & 2983 & 89 \\
\hline $\mathrm{H}(11 \mathrm{C})$ & & 6421 & 4970 & 3259 & 89 \\
\hline $\mathrm{H}(12 \mathrm{~A})$ & & 6708 & 392 & 3586 & 78 \\
\hline $\mathrm{H}(12 \mathrm{~B})$ & & 5192 & 1032 & 3160 & 78 \\
\hline $\mathrm{H}(12 \mathrm{C})$ & & 5422 & 1359 & 4027 & 78 \\
\hline $\mathrm{H}(13)$ & 6309 & 3168 & 1897 & 55 & \\
\hline $\mathrm{H}(14)$ & 9190 & 1785 & 1353 & 55 & \\
\hline $\mathrm{H}(16 \mathrm{~A})$ & & 9054 & 3194 & -303 & 96 \\
\hline $\mathrm{H}(16 \mathrm{~B})$ & & 7219 & 3637 & -215 & 96 \\
\hline $\mathrm{H}(17 \mathrm{~A})$ & & 6803 & 1184 & -284 & 91 \\
\hline $\mathrm{H}(17 \mathrm{~B})$ & & 8595 & 795 & -49 & 91 \\
\hline
\end{tabular}

